\title{
ON THE FOUNDATIONS OF PLANE ANALYSIS SITUS*
}

\author{
BY \\ ROBERT L. MOORE
}

\section{Introduction}

The present paper contains three systems of axioms, $\Sigma_{1}, \Sigma_{2}$, and $\Sigma_{3}$. Each of these systems is a sufficient basis for a considerable body of theorems in the domain of plane analysis situs or what may be roughly termed the nonmetrical part of plane point-set theory, including the theory of plane curves. The axioms of each system are stated in terms of a class of elements called poincs and a class of point-sets called regions.

On the basis of $\Sigma_{1}$ the existence of simple continuous curves is proved as a theorem and it is shown that every region is the interior of a simple closed curve.

$\Sigma_{2}$ is equivalent $\dagger$ to $\Sigma_{1}$ as far as statements in terms of point and limit point are concerned. But $\Sigma_{2}$ is satisfied if in an ordinary euclidean space of two dimensions the term region is interpreted so as to apply to every bounded, connected set of points $R$ of connected exterior such that every point of $R$ lies in the interior of some triangle that is contained in $R$.

Both $\Sigma_{1}$ and $\Sigma_{2}$ contain an axiom (Axiom 1) which postulates the existence of a countable sequence of regions containing a set of subsequences that close down in a specified way on the points of space. Among other things this axiom implies that the set of all points is separable. $\neq$

The set $\Sigma_{3}$ is obtained from $\Sigma_{2}$ by replacing Axioms 1 and 2 by two other axioms, Axioms $1^{\prime}$ and $2^{\prime}$. Here Axiom $1^{\prime}$ postulates the existence for each point $P$ of a countable sequence of regions that closes down on $P$, while Axiom $2^{\prime}$ postulates that every two points of a region are the extremities of at least one arc lying in that region. $\Sigma_{2}$ implies $\Sigma_{3}$ but not conversely.

Though Theorems 1-52 of the present paper are all consequences of $\Sigma_{3}$ nevertheless there exists a space that satisfies $\Sigma_{3}$ but is neither metrical, descriptive§ nor separable. It is interesting that no space that satisfies $\Sigma_{3}$

* A part of this paper was presented to the Society under a different title April 24, 1915. $\dagger$ See $\S 9$.

$\ddagger$ For a definition of the term separable see M. Fréchet, Sur quelques points du calcul fonctionnel, Rendicontidel Circolo Matematico di Palermo, vol. 22 (1906), p. 23.

$\$$ A space $S$ is said to be descriptive or potentially descriptive if it contains a system of open curves (as defined in $\S 8$ ) such that through every two points of $S$ there is one and only one curve of this system. I have not determined whether every space satisfying $\Sigma_{1}$ is potentially descriptive. 
can be potentially descriptive without being separable and, indeed, metrical in the sense that it is in a one to one continuous correspondence with an ordinary euclidean space of two dimensions.

\section{Axioms AND DEFINITIONS}

I consider a class, $S$, of elements called points and a class of sub-classes of $S$ called regions, subject to a set of postulates (axioms) as described below. Before stating these axioms I will define certain terms that will be used.

Definitions. A point $P$ is said to be a limit point of a point-set $M$ if, and only if, every region that contains $P$ contains at least one point of $M$ distinct from $P$. The boundary of a point-set $M$ is the set of all points [ $X$ ] such that every region that contains $X$ contains at least one point of $M$ and at least one point that does not belong to $M$. If $M$ is a point-set, $M^{\prime}$ denotes the set of points composed of $M$ plus its boundary. If $R$ is a region, the point-set $S-R^{\prime}$ is called the exterior of $R$. A point in the exterior of $R$ is said to be without $R$.

A set of points is said to be connected if, however it be divided into two mutually exclusive subsets, one of them contains a limit point of the other one.

A set of regions $K$ is said to cover a point-set $M$ if every point of $M$ belongs to at least one region of the set $K$. If for every infinite set of regions $K$ covering the point-set $M$ there exists a finite subset of $K$ that also covers $M$ then $M$ is said to possess the Heine-Borel property.

Axıom 1.* There exists an infinite sequence of regions, $K_{1}, K_{2}, K_{3}, \cdots$ such that (1) if $m$ is an integer and $P$ is a point, there exists an integer $n$, greater than $m$, such that $K_{n}$ contains $P,(2)$ if $P$ and $\bar{P}$ are distinct points of a region $R$ then there exists an integer $\delta$ such that if $n>\delta$ and $K_{n}$ contains $P$ then $K_{n}^{\prime}$ is a subset of $R-\bar{P}$.

Axiom 2. Every region is a connected set of points.

Axiom 3. If $R$ is a region, $S-R^{\prime}$ is a connected set of points.

Axıom 4. If $R$ is a region, $R^{\prime}$ possesses the Heine-Borel property.

Axıом 5. There exists an infinite set of points that has no limit point.

Axıom 6. If $R$ and $\bar{R}$ are regions and $P$ is a point in $\bar{R}$ and on the boundary of $R$, then there exist in $\bar{R}$ two regions $K$ and $\bar{K}$ such that $\bar{K}$ contains $P, K$ lies in $R$ and all those points of the boundary of $R$ that lie in $\bar{K}$ are points also of the boundary of $K$.

Axiom 7. If $R$ and $\bar{R}$ are regions and $P$ is a point in $\bar{R}$ and on the boundary of $R$, then there exist in $\bar{R}$ two regions $L$ and $\bar{L}$ such that $\bar{L}$ contains $P, L$ lies

* There is a certain amount of resemblance between Axiom 1 and Veblen's Postulate of Uniformity. Cf. O. Veblen, Definition in terms of order alone in the linear continuum and in well-ordered sets, these T r a n s a c t i o $\mathrm{s}$, vol. 6 (1905), p. 169. 
in $S-R^{\prime}$ and all those points of the boundary of $R$ that lie in $\bar{L}$ are points also of the boundary of $L$.

Ахгом 8. Every simple closed curve* is the boundary of at least one region.

\section{Consequences of Axioms $1-3,5, \dagger$}

THEOREM 1. No point of a region is a boundary point of that region.

Theorem 2. If $P$ is a limit point of the point-set $M$ then every region that contains $P$ contains infinitely many points of $M$.

Proof. Suppose the region $R$ contains $P$. By hypothesis $R$ contains at least one point $P_{1}$ which belongs to $M$ and is distinct from $P$. By Axiom 1 there exists in $R$ a region $R_{1}$ containing $P$ but not containing $P_{1}$. The region $R_{1}$ contains at least one point $P_{2}$ belonging to $M$ and distinct from $P$. This process may be continued. It follows that $R$ and $M$ have in common an infinite sequence of distinct points, $P_{1}, P_{2}, P_{3}, \cdots$.

Theorem 3. If $P$ is a point and $M$ is a finite set of points not containing $P$ then there exists a region containing $P$ but containing no point of $M$.

THEOREM 4. Every region contains infinitely many points.

Proof. Suppose $R$ is a region. There exists in $R$ at least one point $O$. By Axiom $5_{1}$ there exist two points $P_{1}$ and $P_{2}$ distinct from each other and from $O$. By Theorem 3 there exists, about $\ddagger P_{1}$, a region $R_{1}$ containing neither $O$ nor $P_{2}$. Suppose $R_{1}^{\prime}$ contains $O$ or $P_{2}$. Then either $O$ or $P_{2}$ is a limit point of $R_{1}$ and therefore, by Axiom 1 and Theorem 2, $R_{1}$ contains a point $Y$ distinct from $P_{1}$. Hence, by Axiom 1, there exists, about $P_{1}$, a region $\bar{R}_{1}$ such that $\bar{R}_{1}^{\prime}$ is a subset of $R_{1}$. It follows that $\bar{R}_{1}^{\prime}$ contains neither $O$ nor $P_{2}$.

Let $K$ denote $\bar{R}_{1}$ or $R_{1}$ according as $R_{1}^{\prime}$ does or does not contain one of the points $O$ and $P_{2}$. Then $S-K^{\prime}=O+M$ where $M$ is a point-set which does not contain $O$. Hence, by Axiom 3 and Theorem 3, $O$ is a limit point of $M$. Hence $R$ contains infinitely many points of $M$.

THEOREM 5. If $P$ is a point then there exists an infinite sequence of regions $R_{1}, R_{2}, R_{3}, \cdots$ such that (1) $P$ is the only point they have in common, (2) for every $n, R_{n+1}$ is a proper subset of $R_{n}$, (3) if $R$ is a region about $P$ then there exists $n$ such that $R_{n}^{\prime}$ is a subset of $R$.

Proof. Let $R_{1}$ denote the first fundamental region $\S$ that contains $P$. Let $R_{2}$ denote the first fundamental region that follows $R_{1}$ in the fundamental

${ }^{*}$ For definition of simple closed curve see $\S 4$.

† Here $5_{1}$ denotes the axiom that there exist at least three points. Of course this proposition is a part of Axiom 5 .

$\ddagger$ In this connection "about" is synonymous with "containing."

$\$$ Select once for all a definite sequence $K_{1}, K_{2}, K_{3}, \cdots$ satisfying the conditions stated in Axiom 1. This definite sequence will be called the fundamental sequence and its regions will be termed fundamental regions. Hereafter in this paper $K_{n}$ denotes the $n$th region in the fundamental sequence: 
sequence, contains $P$ and is a proper subset of $R_{1}$. In general let $R_{n+1}$ denote the first fundamental region that follows $R_{n}$, contains $P$ and is a proper subset of $R_{n}$. It is clear that the sequence $R_{1}, R_{2}, R_{3}, \cdots$, thus defined, satisfies conditions (1), (2), and (3).

THEOREM 6 . If two regions have a point in common, then they have in common at least one region containing that point.

Proof. Suppose the regions $K$ and $L$ have in common the point $P$. Let $R_{1}, R_{2}, R_{3}, \cdots$ denote a sequence of regions satisfying, with respect to the point $P$, conditions (1), (2), and (3) of Theorem 5 . There exist positive integers $m$ and $n$ such that $R_{m}$ is a subset of $K$ and $R_{n}$ is a subset of $L$. The region $R_{m+n}$ is a common subset of $K$ and $L$.

Theorem 7. If $P$ is a limit point of $M+N$ then it is a limit point either of $M$ or of $N$.

Definition. The point $P$ is said to be a sequential limit point of the sequence of points $P_{1}, P_{2}, P_{3}, \cdots$ if and only if for every region $R$ containing $P$ there exists an integer $\delta$ such that if $n>\delta$ then $P_{n}$ lies in $R$.

Theorem 8. If $P$ is a sequential limit point of a sequence of points, $P_{1}$, $P_{2}, P_{3}, \cdots$, then no other point is a limit point of the point-set

$$
P_{1}+P_{2}+P_{3}+\cdots \text {. }
$$

Proof. Suppose $P_{1}+P_{2}+P_{3}+\cdots$ has a limit point $X$ distinct from $P$. By Theorem 3 there exists a region $R$ containing $P$ but not containing $X$. By Theorem 5 there exists a region $K$, containing $P$, such that $K^{\prime}$ is a subset of $R$. There exists $\delta$ such that if $n>\delta$ then $P_{n}$ lies in $K$. But $X$ does not belong to $K^{\prime}$. Hence it is not a limit point of $P_{\delta+1}+P_{\delta+2}+P_{\delta+3}+\cdots$. By Theorem 3 it is not a limit point of $P_{1}+P_{2}+P_{3}+\cdots+P_{n}$. Hence, by Theorem 7 , it is not a limit point of $P_{1}+P_{2}+P_{3}+\cdots$.

Theorem 9. If $P$ is a limit point of $M$ then there exists an infinite sequence of points belonging to $M$ and all distinct from $P$ such that $P$ is the sequential limit point of this sequence.

Proof. Let $R_{1}, R_{2}, R_{3}, \cdots$ denote an infinite sequence of regions satisfying, with respect to $P$, conditions (1), (2), and (3) of Theorem 5. For every $n, R_{n}$ contains at least one point of $M$ distinct from $P$. With the help of Zermelo's postulate it follows that there exists an infinite sequence of points $P_{1}, P_{2}, P_{3}, \cdots$ such that $P_{n}$ belongs to $R_{n}$ and to $M$ and is distinct from $P$. It is clear that $P$ is the sequential limit point of this sequence.

Definition. If $A$ and $B$ are distinct points, then a simple chain from $A$ to $B$ is a finite sequence of regions $R_{1}, R_{2}, R_{3}, \cdots R_{n}$ such that (1) $R_{i}$ contains $A$ if and only if $i=1$, (2) $R_{i}$ contains $B$ if and only if $i=n$, (3) if $1 \leqq i \leqq n$, $1 \leqq j \leqq n, i<j$ then $R_{i}$ has a point in common with $R_{j}$ if and only if

$$
j=i+1 \text {. }
$$


The region $R_{k}(1 \leqq k \leqq n)$ is said to be the $k$ th link of the chain

$$
R_{1} R_{2} R_{3} \cdots R_{n} \text {. }
$$

Theorem 10. If $M$ is a connected set of points, $A$ and $B$ are two distinct points of $M$ and $G$ is a set of regions covering $M$ then there exists a simple chain from $A$ to $B$ every link of which is a region of the set $G$.

Proof. If there is no such chain from $A$ to $B$ then the points of $M$ can be divided into two classes $S_{A}$ and $S_{B}$, where $S_{A}$ is the set of all points [ $P$ ] such that $P$ can be joined to $A$ by a simple chain every link of which is a region of the set $G$, and $S_{B}$ is the set of all other points of $M$. Since $M$ is connected, one of the sets $S_{A}$ and $S_{B}$ must contain a point $X$ which is a limit point of the other one. The point $X$ lies in at least one region $R$ of the set $G$. The region $R$ contains a point $A_{1}$ belonging to $S_{A}$ and a point $B_{1}$ belonging to $S_{B}$, where $A_{1}$ is $X$ or $B_{1}$ is $X$ according as $X$ belongs to $S_{A}$ or to $S_{B}$. The point $A$ can be joined to $A_{1}$ by a simple chain $R_{1} R_{2} R_{3} \cdots R_{n}$ every link of which is a region of the set $G$. Let $R_{k}$ be the first link of this chain that intersects* $R$. Then $R_{1} R_{2} R_{3} \cdots R_{k} R$ is a simple chain from $A$ to $B_{1}$, every link of which belongs to $G$. Thus the supposition that Theorem 10 is false leads to a contradiction.

Definition. A set of points is said to be closed if it contains all its limit points. A set of points $M$ is said to be bounded if there exists a finite set of regions, $R_{1}, R_{2}, R_{3}, \cdots R_{n}$ such that $M$ is a subset of

$$
\left(R_{1}+R_{2}+R_{3}+\cdots+R_{n}\right)^{\prime} \text {. }
$$

It is clear that if $M$ is a set of points then $M^{\prime}$ is closed.

\section{Consequences of Axioms 1-4, 5}

THEOREM 11. If $R_{1}, R_{2}, R_{3}, \cdots R_{n}$ is a finite set of regions, the point-set $\left(R_{1}+R_{2}+R_{3}+\cdots+R_{n}\right)^{\prime}$ possesses the Heine-Borel property.

Theorem 12. Every closed, bounded set of points possesses the Heine-Borel property.

Proof. Suppose that $R_{1}, R_{2}, R_{3}, \cdots R_{n}$ is a finite set of regions and that $M$ is a closed point-set lying in $\left(R_{1}+R_{2}+R_{3}+\cdots+R_{n}\right)^{\prime}$ and covered by a set of regions $G$. Let $H$ denote the point-set $R_{1}+R_{2}+R_{3}+\cdots+R_{n}$. If every point of $H^{\prime}$ belongs to $M$ then, by Theorem 11, $M$ has the HeineBorel property. Suppose that not every point of $H^{\prime}$ belongs to $M$. Since $M$ is closed therefore about each point of $H^{\prime}-M$ there is a region containing no point of $M$. Thus there exists a set of regions $\bar{G}$ covering $H^{\prime}-M$ but such that no point of $M$ belongs to any region of $\bar{G}$. By Theorem 11 there

\footnotetext{
* Two point-sets are said to intersect each other if they have at least one point in common.
} 
exists a finite set of regions $G_{1}$ covering $H$ and such that each region of $G_{1}$ belongs either to $G$ or to $\bar{G}$. Let $G_{2}$ denote the set of all those regions of $G_{1}$ that intersect $M$. No region of $G_{2}$ can belong to $\bar{G}$. Hence every region of $G_{2}$ belongs to $G$. Moreover $G_{2}$ covers $M$.

Theorem 13. Every infinite, bounded set of points has at least one limit point.

For a proof of Theorem 13 see F. Hausdorff, Grundzüge der Mengenlehre,* page 231 .

Theorem 14. If $M_{1}, M_{2}, M_{3}, \cdots$ is an infinite sequence of bounded point-sets such that, for every $n, M_{n}$ contains $M_{n+1}^{\prime}$, then the point-sets $M_{1}, M_{2}$, $M_{3}, \cdots$ have at least one point in common and the set, $G$, of all such common points is closed.

Proof. For each $n, M_{n}$ contains at least one point. Hence, by Zermelo's postulate, there exists a sequence of points $P_{1}, P_{2}, P_{3}, \cdots$ such that, for each $n, P_{n}$ lies in $M_{n}$. If there exists a positive integer $\bar{n}$ such that, for every $n$ greater than $\bar{n}, P_{n}=P_{\bar{n}}$, then $P_{\bar{n}}$ is common to all the point-sets $M_{1}, M_{2}$, $M_{3}, \cdots$. If no such $\bar{n}$ exists then the sequence $P_{1}, P_{2}, P_{3}, \cdots$ contains infinitely many distinct points and therefore, by Theorem 13 , there exists a point $P$ which is a limit point of $P_{1}+P_{2}+P_{3}+\cdots$. Since $P$ is a limit point of $\left(P_{1}+P_{2}+P_{3}+\cdots+P_{n}\right)+\left(P_{n+1}+\cdots\right)$, therefore, by Theorems 7 and 3 , it is a limit point of $P_{n+1}+\cdots$. But $P_{n+1}+\cdots$ is a subset of $M_{n+1}$, and $M_{n+1}^{\prime}$ is a subset of $M_{n}$. Therefore every $M_{n}$ contains $P$. Hence the set $G$ exists.

Suppose that $O$ is a limit point of $G$. Then, for every $n, O$ belongs to $M_{n+1}^{\prime}$ and therefore to $M_{n}$. Hence $O$ belongs to $G$. Thus $G$ is closed.

Definition. A domain is a connected set of points $M$ such that if $P$ is a point of $M$ then there exists a region that contains $P$ and lies in $M$.

Definition. $\dagger$ If $A$ and $B$ are two distinct points, a simple continuous arc from $A$ to $B$ is a bounded, closed, connected set of points containing $A$ and $B$ but containing no connected proper subset that contains both $A$ and $B$.

Theorem 15. If $A$ and $B$ are distinct points of a domain $M$, there exists $a$ simple continuous arc from $A$ to $B$ that lies wholly in $M$.

Proof. If $P$ is a point of $M, P$ lies in a region $R$ which is contained in $M$.

*Veit \& Co., Leipzig, 1914. See also E. W. Chittenden, The converse of the HeineBorel theorem in a Riesz domain, Bulletin of the A m erican Math e matical S o c i e t y , vol. 21 (1915), pp. 179-183, and vol. 20 (1914), p. 461. For a proof that in the presence of certain linear order postulates the Heine-Borel Theorem is equivalent to the Dedekind-cut Postulate see O. Veblen, The Heine-Borel Theorem, Bulletin of the American Mathematical Socicty, vol. 10 (1903-04) pp. 436-439.

$\dagger$ See N. J. Lennes, Curves in non-metrical analysis situs with an application in the calculus of variations, American Journal of Mathematics, vol. 33 (1911), page 308, and Bulletin of the American Mathematical Society, vol. 12 (1906), p. 284. 
There exists $n_{1 P}$ greater than or equal to 1 such that $K_{n_{1 P}}$ contains $P$ and such that if $n \geqq n_{1 P}$ and $K_{n}$ contains $P$ then $K_{n}^{\prime}$ is contained in $R$ and therefore in $M$. Let $G_{1}$ be the set of all such $K_{n_{1 P}}$ 's for all $P$ 's in $M$. By Theorem 10 there exists a simple chain $R_{11} R_{12} \cdots R_{1 m_{1}}$, from $A$ to $B$, every link of which belongs to $G_{1}$. Call this chain $C_{1}$. For each $i\left(1 \leqq i<m_{1}\right)$ select a point $P_{1 i}$ common to $R_{1 i}$ and $R_{1 i+1}$. Let $P_{10}=A$ and $P_{1 m_{1}}=B$. If $0 \leqq i<m_{1}$ then $P_{1 i}$ can be joined to $P_{1 i+1}$ by a simple chain $C_{1 i+1}$ each link of which is a region of the sequence $K_{2}, K_{3}, \cdots$ and lies, with all its limit points, in $R_{1 i+1}$. If for any $i\left(1 \leqq i \leqq m_{1}\right)$ any link except the last one of the chain $C_{1 i}$ intersects any link of $C_{1 i+1}$ then omit from $C_{1 i}$ every link that follows $\bar{R}_{1 i}$ where $\bar{R}_{1 i}$ is the first link of $C_{1 i}$ that intersects a link of $C_{1 i+1}$; also omit from $C_{1 i+1}$ every link (if there be any such) that precedes the last link that intersects $\bar{R}_{1 i}$. These omissions having been made for each $i$ concerned, the remaining links of the chains $C_{11}, C_{12}, \cdots C_{1 m_{1}}$ form a simple chain $C_{2}$ from $A$ to $B$. The chain $C_{2}$ has the important property that each one of its links lies wholly in some single link of the chain $C_{1}$ and if a link $x$ of $C_{2}$ lies in a link $y$ of $C_{1}$ then every link that follows $x$ in $C_{2}$ lies either in $y$ or in some link that follows $y$ in $C_{1}$. Similarly there exists a chain $C_{3}$ having a relation to $C_{2}$ analogous to the above indicated relation of $C_{2}$ to $C_{1}$ and such that every link of $C_{3}$ is a region of the sequence $K_{3}, K_{4}, \cdots$. This process may be continued. Thus there exists an infinite sequence of chains $C_{1}, C_{2}$, $C_{3} \ldots$ such that (1) each link of the chain $C_{n+1}$ lies, together with all its limit points, wholly in some single link of $C_{n}$; (2) if a link $x$ of $C_{n+1}$ lies in a link $y$ of $C_{n}$ then each link that follows $x$ in $C_{n+1}$ lies either in $y$ or in some link that follows $y$ in $C_{n} ;(3)$ every link of $C_{n}$ is a region of the sequence $K_{n}, K_{n+1}$, * $K_{n+2}, \cdots$.

Let $\bar{C}_{n}$ denote the point-set which is the sum of all the links of the chain $C_{n}$. Let $C$ denote the set of all those points that the sets $\bar{C}_{1}, \bar{C}_{2}, \bar{C}_{3}, \cdots$ have in common. It will be proved that $C$ satisfies Lennes' definition of a Jordan arc from $A$ to $B$.

I. To prove that $C$ is closed. Every limit point of a single link of $C_{n+1}$ lies in some link of $C_{n}$. Hence, by Theorem $7, \bar{C}_{n+1}^{\prime}$ is a subset of $\bar{C}_{n}$. It follows by Theorem 14 that $C$ is closed.

II. To prove that $C$ is connected. Suppose that $C$ can be divided into two mutually exclusive subsets $S_{1}$ and $S_{2}$ neither of which contains a limit point of the other one. Since $C$ is closed every limit point of $S_{1}$ belongs to $S_{1}+S_{2}$ and therefore must belong to $S_{1}$. Hence $S_{1}$ is closed. Likewise $S_{2}$ is closed. About each point $P$ of $S_{1}$ there is a region $R$ containing no point of $S_{2}$. There exists $\bar{n}$ such that $K_{\bar{n}}$ contains $P$ while $K_{\bar{n}}^{\prime}$ lies in $R$. Hence, by Theorem 10, there exists a finite set $G_{1}$ of regions such that every point of $S_{1}$ belongs to some region of $G_{1}$ but no point of $S_{2}$ belongs to; or is a limit point of, any 
region of $G_{1}$. Since the set $G_{1}$ is finite it follows, by Theorem 7 , that no point of $S_{2}$ is a limit point of $\bar{G}_{1}$ (the point-set which is the sum of all the regions of the set $G_{1}$ ). About each point of $S_{2}$ there is a region such that no point of $\bar{G}_{1}^{\prime}$ is a point or a limit point of that region. Hence there exists a finite set $G_{2}$ of regions, covering $S_{2}$ and such that no point of $\bar{G}_{1}^{\prime}$ is a point or a limit point of any region of $G_{2}$. Let $\bar{G}_{2}$ denote the point-set which is the sum of all the regions of the set $G_{2}$. Then the two closed point-sets $\bar{G}_{1}^{\prime}$ and $\bar{G}_{2}^{\prime}$ have no point in common. But $\bar{C}_{n}$ contains at least one point of $\bar{G}_{1}^{\prime}$ and at least one point of $\bar{G}_{2}^{\prime}$. Hence, since $\bar{C}_{n}$ is connected it must contain at least one point that does not belong to $\bar{G}_{1}^{\prime}+\bar{G}_{2}^{\prime}$. Let $T_{n}$ denote the set of all such points. For every $n, T_{n+1}^{\prime}$ is a subset of $T_{n}$. Hence, by Theorem 14, the point-sets $T_{1}, T_{2}, \cdots$ have in common at least one point $O$. The point $O$ belongs to $C$ and therefore to $\bar{G}_{1}+\bar{G}_{2}$ as well as to $T_{1}$. Thus the supposition that $C$ is not connected leads to a contradiction.

III. To prove that no connected proper subset of $C$ contains both $A$ and $B$. Let us first order the points of $C$. If $X_{1}$ and $X_{2}$ are two distinct points of $C$ then there exists $n$ such that $X_{1}$ and $X_{2}$ do not both lie in the same region of the set $K_{n}, K_{n+1}, K_{n+2}, \cdots$. But every link of $C_{n}$ is a region of this set. Thus for every two distinct points $X_{1}$ and $X_{2}$ belonging to $C$ there exists $n$ such that $X_{1}$ and $X_{2}$ do not belong to the same link of $C_{n}$. Furthermore it is clear that if $X_{1}$ and $X_{2}$ do not lie in the same link of $C_{n}$ but $X_{1}$ lies in a link of $C_{n}$ that precedes one in which $X_{2}$ lies, then if $m>n$ every link of $C_{m}$ that contains $X_{1}$ precedes every link of $C_{m}$ that contains $X_{2}$. The point $X_{1}$ is said to precede the point $X_{2}\left(X_{1}<X_{2}\right)$ if there exists $n$ such that every link of $C_{n}$ that contains $X_{1}$ precedes every link of $C_{n}$ that contains $X_{2}$. From facts observed above it follows that if $X_{1}$ and $X_{2}$ are distinct points of $C$ then either $X_{1}<X_{2}$ or $X_{2}<X_{1}$, while if $X_{1}<X_{2}$ then it is not true that $X_{2}<X_{1}$. Furthermore if $X_{1}<X_{2}$ and $X_{2}<X_{3}$ then $X_{1}<X_{3}$. For there exist $n_{1}$ and $n_{2}$ such that $X_{1}$ and $X_{2}$ do not lie in the same link of $C_{n_{1}}$ and $X_{2}$ and $X_{3}$ do not lie in the same link of $C_{n_{2}}$. Hence every link of $C_{n_{1}+n_{3}}$ that contains $X_{1}$ precedes every link of $C_{n_{1}+n_{2}}$ that contains $X_{2}$ and every link of $C_{n_{1}+n_{2}}$ that contains $X_{2}$ precedes every one that contains $X_{3}$. Hence every one that contains $X_{1}$ precedes every one that contains $X_{3}$. Hence $X_{1}<X_{3}$.

Suppose now that $H$ is a proper subset of $C$ that contains both $A$ and $B$. Then there exists a point $P$ belonging to $C$ but different from $A$ and from $B$ such that $H$ is a subset of $C-P$. Now $C-P=S_{A}+S_{B}$ where $S_{A}$ is the set of all points of $C$ that precede $P$ and $S_{B}$ is the set of all points of $C$ that follow $P$. It is clear that $S_{A}$ contains $A$ and $S_{B}$ contains $B$. Suppose that $P_{A}$ is a point of $S_{A}$. Then there exists $n$ such that every link of $C_{n}$ that contains $P_{A}$ precedes every one that contains $P$. Suppose that some link $y$ of 
the chain $C_{n}$ contains $P_{A}$ and also a point $P_{B}$ of the set $S_{B}$. Since $y$ precedes every link of $C_{n}$ that contains $P$ it follows that $P_{B}$ precedes $P$, which is contrary to hypothesis. Hence no region of $C_{n}$ that contains $P_{A}$ contains any point of $S_{B}$. But some region of $C_{n}$ does contain $P_{A}$. Hence $P_{A}$ is not a limit point of $S_{B}$. Similarly no point of $S_{B}$ is a limit point of $S_{A}$. But $H$ contains a point $A$ that belongs to $S_{A}$ and a point $B$ that belongs to $S_{B}$. Moreover $H$ is a subset of $S_{A}+S_{B}$. It follows that $H$ is not connected.

It is thus established that $C$ is a simple continuous arc from $A$ to $B$.

Theorem 16. Every two points of a region $R$ can be joined by an arc* lying entirely in $R$ while every two points without $R$ can be joined by an arc lying entirely without $R$.

\section{Consequences of Axioms 2-5 and Theorem 5}

On the basis of Axioms 2-5 and Theorem 5 it is possible to prove Theorems $2-9$ of $\S 4$ of Lennes' paper. $\dagger$ In particular if $X$ is any point of the arc $A B$ then $A B$ is the sum of two $\operatorname{arcs} A X$ and $X B$ that have no common point other than $X$. If $X$ and $Y$ are points of the arc $A B$ distinct from $A$ and from $B$ then $A B$ contains as a subset only one arc that has $X$ and $Y$ as its endpoints. This arc $X Y$ is called the interval $X Y$ of the arc $A B$. If $Z$ is a point (distinct from $X$ and from $Y$ ) of the interval $X Y$ of the $\operatorname{arc} A B$ then $Z$ is said to be between $X$ and $Y$ on the arc $A B$. If $X$ is between $A$ and $Y$ on the arc $A B$ then $X$ is said to precede $Y$ on $A B$ and $Y$ is said to follow $X$ on $A B$. If $X$ precedes $Y$ on $A B$ then $Y$ precedes $X$ on $B A$.

If the arc $A B$ has at least one point in common with the closed set of points $K$ then there exists (1) a point $P_{1}$ common to $A B$ and $K$ such that if $X_{1}$ is common to $A B$ and $K$ and $X_{1} \neq P_{1}$ then $X_{1}$ is between $P_{1}$ and $B$ on the arc $A B$, (2) a point $P_{2}$ common to $A B$ and $K$ such that if $X_{2}$ is common to $A B$ and $K$ and $X_{2} \neq P_{2}$ then $X_{2}$ is between $P_{2}$ and $A$ on the arc $A B$. The point $P_{1}$ is said to be the first point that $A B$ has in common with $K$ while $P_{2}$ is said to be the last point that $A B$ has in common with $K$. It is to be observed that the first point that $A B$ has in common with $K$ is the last point that $B A$ has in common with $K$.

If $P$ is a point on an arc $A B$ and $M$ is a point-set on $A B$ then $P$ is a limit point of $M$ if and only if every interval of $A B$ that contains $P$ (but does not have $P$ as an endpoint) contains also a point of $M$ distinct from $P$.

Definition. $\ddagger$ A simple closed curve is a set of points composed of two arcs $A X B$ and $A Y B$ that have no point in common except $A$ and $B$.

* Hereafter in this paper, "arc" and "simple continuous arc" will be considered synonymous terms.

$\dagger$ Loc. cit.

† See N. J. Lennes, loc. cit., p. 314. 
If $A$ and $B$ are two distinct points of the simple closed curve $J$ then there are two and only two distinct arcs of $J$ from $A$ to $B$. These two arcs, $A X B$ and $A Y B$, have no point in common except their endpoints, $A$ and $B$. Every point of $J$ belongs either to $A X B$ or to $A Y B$ and $A$ and $B$ are said to separate $J$ into the two arcs $A X B$ and $A Y B$. If $A, B, C$, and $D$ are four distinct points of $J$ such that $C$ lies on one and $D$ lies on the other of the two arcs into which $A$ and $B$ separate $J$ then $A$ and $B$ are said to separate $C$ and $D$ on $J$. If $A$ and $B$ separate $C$ and $D$ on $J$ then $C$ and $D$ separate $A$ and $B$ on $J$.

ThEOREM 17. If $R$ is a region, the exterior of $R$ contains an infinite set of points that has no limit point.

Theorem 17 is a consequence of Axiom 5 and Theorem 13.

THEOREM 18. The exterior of a region is not a bounded point-set.

Theorem 19. Every region has at least one boundary point.

Proof. Suppose $R$ is a region. By Theorems 4 and 5 there exist in $R$ two points $P$ and $X$ and a region $R_{n}$ such that $R_{n}$ contains $P$ but $R_{n}^{\prime}$ is a subset of $R-X$. It follows by Theorem 17 that $S-R_{n}^{\prime}=M_{1}+M_{2}$ where $M_{1}$ is a subset of $R$ and $M_{2}$ is a subset of $S-R$. No point of $M_{1}$ is a limit point of $M_{2}$. Hence, by Axiom 3, $M_{2}$ contains a limit point of $M_{1}$. Every such point is a boundary point of $R$

\section{Consequences of Axioms 2-8 and Theorems 5 and 15}

It is clear that Theorems 1-19 are consequences of Axioms 2-5 and Therems 5 and 15 .

TheOREm 20. Every point of the boundary of a region is a limit point of the exterior of that region.

Theorem 20 can easily be proved with the help of Axiom 7.

TheOREm 21. If $K$ and $R$ are regions and the boundary of $R$ is a subset of $K^{\prime}$ then $R$ is a subset of $K$.

Proof. By Theorem 18, $S-K^{\prime}$ contains at least one point that does not belong to $R$. If it contains also a point of $R$ then $S-K^{\prime}=S_{1}+S_{2}$ where $S_{1}$ is a subset of $R$ but no point of $S_{2}$ belongs to $R$. The point-set $S_{1}$ cannot contain a limit point of $S_{2}$. Hence, by Axiom $3, S_{2}$ must contain a limit point of $S_{1}$. Hence $S_{2}$ contains a point of the boundary of $R$. But this is contrary to hypothesis. It follows that $R$ is a subset of $K^{\prime}$. If $R$ contained a point on the boundary of $K$ then, by Theorem 20, it would contain a point of $S-K^{\prime}$. It follows that $R$ is a subset of $K$.

THEOREM 22. The set of all points is connected.

Theorem 22 can be easily proved with the help of Theorem 19, Axioms 2 and 3 and Theorem 20.

THEOREM 23. No region is a subset of an arc.

Proof. Suppose that the region $R$ is a subset of the arc $A B$. Then $R$ 
must contain at least one point $O$ belonging to $A B$ but distinct from $A$ and from $B$. By Theorems 3 and 5 there exists about $O$ a region $K$ such that $A$ and $B$ are both without $K$. By Theorem 16 there exists an arc $A X B$ lying without $K$. There exist on $A B$ two points $A_{1}$ and $B_{1}$ sucb that $A_{1}$ is the last point that $A X B$ has in common with the interval $A O$ of the arc $A B$ and $B_{1}$ is the first point following $A_{1}$ on $A X B$ that $A X B$ has in common with the interval $O B$ of the arc $A B$. The arc $A_{1} O B_{1}$ on $A B$ and the arc from $B_{1}$ to $A_{1}$ on $A X B$ constitute together a closed curve $\bar{J}$. There exists about $O$ a region $R_{1}$ containing no point of the closed point-set $A A_{1}+B_{1} B$, where $A A_{1}$ and $B B_{1}$ are intervals of $A B$. By Theorem 6 the regions $R$ and $R_{1}$ contain in common a region $R_{2}$ that contains $O$. Since $R_{2}$ is a subset of $R$ that contains no point of $A A_{1}+B_{1} B$ it must be a subset of $A_{1} O B_{1}$ and therefore of $\bar{J}$. But $O$ is on the boundary of $\bar{R}$, the interior of $\bar{J}$. Therefore, since $R_{2}$ contains $O, R_{2}$ must contain a point of $\bar{R}$. Thus the supposition that $R$ is a subset of $A B$ leads to a contradiction.

Theorem 24. If the points $A$ and $B$ separate the points $C$ and $D$ on the closed curve* $J$ and $A X B$ is an arc such that $A X B \dagger$ is a subset of $R$, the interior of $J$, then (1) $R_{1}$, the interior of the closed curve $A X B C A$, is a subset of $R$, (2) $A D B$ is entirely without $R_{1}$, (3) $R_{1}$ has no point in common with $R_{2}$, the interior of $A X B D A$.

Proof. That $R_{1}$ is a subset of $R$ is a consequence of Theorem 21. Hence, by Theorem $1, R_{1}$ contains no point of $A D B$.

Suppose that $R_{1}$ and $R_{2}$ have a point in common. Since the boundary of $R_{2}$ contains points that are neither in $R_{1}$ nor on the boundary of $R_{1}, R_{2}$ is not a subset of $R_{1}$. Hence $R_{2}=S_{1}+S_{2}$ where $S_{1}$ is a subset of $R_{1}$ but no point of $S_{2}$ belongs to $R_{1}$. The point-set $S_{1}$ cannot contain a limit point of $S_{2}$. Hence, by Axiom 2, $S_{2}$ must contain a point $P$ which is a limit point of $S_{1}$. Clearly $P$ must be on the boundary of $R_{1}$. Thus $R_{2}$ would contain a point of $A X B$ or of $A C B$. But this is contrary to hypothesis and (2). It follows that $R_{1}$ and $R_{2}$ have no point in common.

TheOREM 25. Under the same hypothesis as in Theorem 24,

$$
R=\underline{A X B}+R_{1}+R_{2} .
$$

Proof. Suppose it is not true that $R=A X B+R_{1}+R_{2}$. Then, by hypothesis and Theorem $24, R=A X B+R_{1}+R_{2}+Y$ where the pointsets $A X B, R_{1}, R_{2}$, and $Y$ are mutually exclusive. It is clear that no one of the three sets $R_{1}, R_{2}$, and $Y$ contains a limit point of either of the other

* Hereafter in this paper, "closed curve" will be considered synonymous with "simple closed curve."

$\dagger$ If $A x B$ is an arc, $A X B$ denotes the point-set $A X B-A-B$. Likewise if $A B$ is an arc, $A B$ denotes the point-set $A B-A-B$. 
two. Let $E$ denote a point of $Y$. By Theorem 16 there exists an arc $E X$ lying entirely in $R$. There exists a point $O$ (Fig. 1) which is the first point that $E X$ has in common with $A X B$. Let $O E$ denote the interval of $E X$ whose endpoints are $O$ and $E$. Now, (1) $O E-O$ is connected, (2) every point of $O E-O$ belongs to $R_{1}$ or to $R_{2}$ or to $Y$, (3) no one of the sets $R_{1}, R_{2}$, and $Y$ contains a limit point of either of the other two. It follows that since $E$ be-

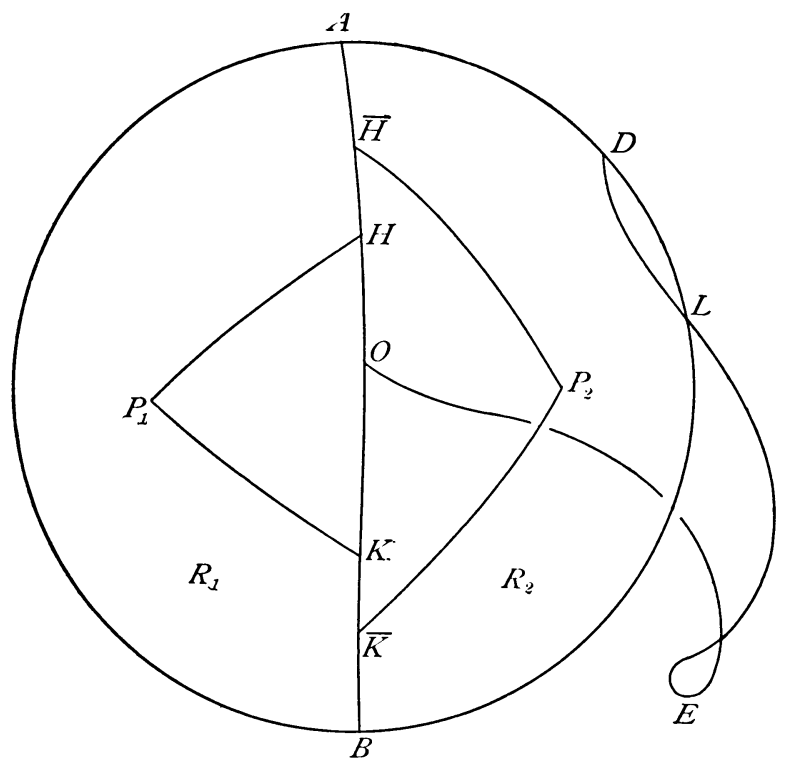

FIG. 1

longs to $Y$ therefore $O E-O$ is a subset of $Y$. Let $F$ denote a point of $R_{1}$. The point $O$ divides the arc $A X B$ into two arcs $A \bar{A} O$ and $B \bar{B} O$. There exists about $\bar{A}$ a region $T$ which contains no point of the closed point-set $F+O \bar{B} B+J$. The region $T$ contains at least one point $G$ in common with $R_{1}$. By Theorem 16 there is an arc $F G$ lying entirely in $R_{1}$ and an arc $G \bar{A}$ lying entirely in $T$. It can easily be shown that the point-set $F G+G \bar{A}$ contains as a subset an $\operatorname{arc} F H$ such that $H$ is in $T$ and on the boundary of $R_{1}$ (and therefore on $A \bar{A} O$ ) while $F H-H$ is a subset of $R_{1}$. Similarly there exists an arc $F K$ such that $K$ is a point of $O \bar{B} B$ and $F K-K$ is a subset of $R_{1}$. The point-set $F H+F K$ contains as a subset an arc $H P_{1} K$. Clearly $H P_{1} K$ is a subset of $R_{1}$. Likewise there exist a point $\bar{H}$ between $A$ and $H$, a point $\bar{K}$ between $B$ and $K$ and an arc $\bar{H} P_{2} \bar{K}$ such that $\bar{H} P_{2} \bar{K}$ is a subset of $R_{2}$. Let $\bar{J}$ denote the closed curve $\bar{H} P_{2} \bar{K}+\bar{K} K+K P_{1} H+H \bar{H}$. By Theorem 21, $\bar{R}$ (the interior of $\bar{J}$ ) is a subset of $R$. But $P_{1}$ is on $\bar{J}$ and is therefore a limit point of $\bar{R}$. Moreover $P_{1}$ belongs to $R_{1}$ and no point of $R_{1}$ is a limit point of $A X B$ or of $R_{2}$ or of $Y$. It follows that $\bar{R}$ must contain at least one point 
of $R_{1}$. Similarly $P_{2}$ is a limit point of $\bar{R}$ and $\bar{R}$ must contain at least one point of $R_{2}$. Since $D$ and $E$ are without the region $R_{1}$ therefore there exists an arc $D E$ lying entirely without $R_{1}$. There exists on $A D B$ a point $L$ wbich is the first point that $E D$ has in common with $A D B$. The point-set $E L-L$ lies in $R$ and contains no point of $A X B$. It easily follows that $E L-L$ is a subset of $Y$. But $E O-O$ is a subset of $Y$. It follows that there exists an $\operatorname{arc} O L$ such that $O L$ is a subset of $Y$. Hence $O L$ contains no point of $\bar{J}$. But $L$ is witbout $\bar{J}$. Therefore $O$, and consequently $H O K$, is without $\bar{J}$. Hence every point of $\bar{R}$ belongs to $R_{1}, R_{2}$, or $Y$. But no one of these three point-sets contains a limit point of either of the other two and it has been shown that $\bar{R}$ contains at least one point of $R_{1}$ and at least one point of $R_{2}$. Hence $\bar{R}$ is not connected. But this is contrary to Axiom 2. Thus the supposition that $Y$ exists leads to a contradiction. It follows that

$$
R=R_{1}+R_{2}+\underline{A X B} .
$$

Theorem 26. If $A$ and $B$ are distinct points and $A C B, A D B$, and $A F B$ are three arcs no two of which have any point in common except $A$ and $B$, then the regions which are bounded by the closed curves $A C B F A, A C B D A$, and $A D B F A$ respectively are not mutually exclusive.

Proof. Denote the curves $A C B F A, A C B D A$, and $A D B F A$ by $J, J_{1}, J_{2}$, their interiors by $R, R_{1}, R_{2}$ and their exteriors by $E, E_{1}, E_{2}$ respectively.

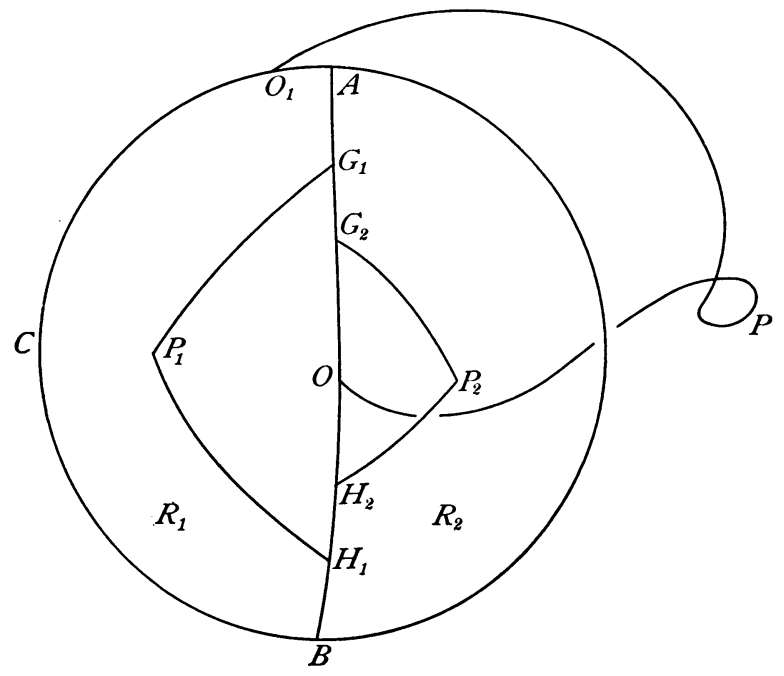

FIG. 2

Suppose that $R, R_{1}, R_{2}$ are mutually exclusive. Then $E$ contains $A D B, R_{1}$, and $R_{2}$. It may be easily proved with the assistance of Theorems 17 and 13 that $E$ contains at least one point which does not belong to $R_{1}, R_{2}$, or $A D B$. 
Let $Y$ denote the set of all such points. It is clear that $Y$ is composed of all points that are common to $E, E_{1}$, and $E_{2}$. Let $P$ (Fig. 2) be a point of $Y$. There exists an arc $P D$ which lies entirely in $E$. Let $O$ be the first point that $P D$ has in common with $A D B$. Then $P O-O$ is clearly a subset of $Y$. Similarly there exists an arc $\overline{P U_{1}}$ such that $O_{1}$ is a point on $A C B$ and such that $P O_{1}-O_{1}$ is a subset of $Y$. It can easily be shown* that there exist points $G_{1}, G_{2}, H_{1}, H_{2}$ lying on $A D B$ in the order $A G_{1} G_{2} O H_{2} H_{1} B$ and arcs $G_{1} P_{1} H_{1}, G_{2} P_{2} H_{2}$ such that $G_{1} P_{1} H_{1}$ and $G_{2} P_{2} H_{2}$ lie in $R_{1}$ and $R_{2}$ respectively. Let $\bar{J}, \bar{J}_{1}, \bar{J}_{2}$ denote the closed curves $G_{1} P_{1} H_{1} H_{2} P_{2} G_{2} G_{1}$, $G_{1} P_{1} H_{1} O G_{1}, G_{2} P_{2} H_{2} O G_{2}$ respectively. Let $\bar{R}, \bar{R}_{1}, \bar{R}_{2}$ denote their respective interiors. It follows from Theorem 21 that $\vec{R}_{1}$ and $\vec{R}_{2}$ are subsets of $R_{1}$ and $R_{2}$ respectively.

There are three cases to be considered.

Case I. Suppose that $O$ lies in $\bar{R}$. Then, by Theorem 25,

$$
R=\bar{R}_{1}+\bar{R}_{2}+\mathrm{G}_{2} \mathrm{OH}_{2} .
$$

It follows that if $O$ is a limit point of a point set $M$ then $M$ must contain a point of $\bar{R}_{1}$ or of $\bar{R}_{2}$ or of $G_{2} O H_{2}$. But $O$ is a limit point of $P O-O$ and $P O-O$ contains no point of $\bar{R}_{1}+\bar{R}_{2}+G_{2} O H_{2}$. Thus the supposition that $O$ is in $\bar{R}$ leads to a contradiction.

Case II. Suppose that $A$ is in $\vec{R}$. Then $J$ lies in $\vec{R}$. But $O_{1}$ is a point of $J$ and $P O_{1}$ contains no point of $\bar{J}$. Hence $P$ is in $\bar{R}$. Similarly every other point of $Y$ is in $\bar{R}$. But $Y$ contains an infinite set of points that has no limit point. Hence, by Theorem $13, Y$ is not a subset of $\bar{R}$. Thus the supposition that $A$ is in $\bar{R}$ leads to a contradiction.

Case III. Suppose that neither $O$ nor $A$ is in $\bar{R}$. Then no point of $J$, or of $A D B$ is in $\bar{R}$. Hence $\bar{R}$ is a subset of $R+R_{1}+R_{2}+Y$. But $\bar{R}$ is connected and no one of the point-sets $R_{1}, R_{2}, R$, and $Y$ contains a limit point of one of the others. Hence $\bar{R}$ cannot contain a point of one of these pointsets and also a point of one of the others. Therefore $\vec{R}$ must be a subset of either $R, R_{1}, R_{2}$, or $Y$. But $P_{1}$ and $P_{2}$ are both limit points of $\bar{R}$ and moreover $P_{1}$ is a point of $R_{1}$ and $P_{2}$ is a point of $R_{2}$. Hence either (1) $R_{1}$ contains a limit point of $R, R_{2}$, or $Y$ or (2) $R_{2}$ contains a limit point of $R_{1}$. Thus the supposition that $\bar{R}$ contains neither $O$ nor $A$ leads to a contradiction.

It follows that $R, R_{1}$, and $R_{2}$ are not mutually exclusive.

Theorem $2 \%$. If the points $A$ and $B$ separate the points $C$ and $D$ on the closed curve $J$ and $A X B$ is an arc such that $A X B$ is without $J$ then (1) either $D$ is without $A X B C A$ or $C$ is without $A X B \overline{D A,(2)}$ If $D$ is without $A X B C A$ then $C$ is within $A X B D A$ and the interior of $A X B D A=A C B+$ the interior of $A C B D A+$ the interior of $A X B C A$.

* Cf. proof of Theorem 25. 
Proof. (1) Suppose $D$ is not without $A X B C A$. Then $A D B$ is within $A X B C A$. Hence, by Theorem $24, C$ is without $A X B D A$.

(2) Suppose that $D$ is without $A X B C A$. Then $A D B$ is without $A X B C A$. Let $R$ and $R_{1}$ denote the interiors of $A C B D A$ and $A X B C A$ respectively. Suppose that $R$ and $R_{1}$ have a point in common. Then $R_{1}=M+M_{1}$ where $M$ is a subset of $R$ but no point of $M_{1}$ belongs to $R^{\prime}$. Neither of the point-sets $M$ and $M_{1}$ can contain a limit point of the other one. Thus Axiom 2 is contradicted. It follows that if $D$ is without $A X B C A$ then the interiors of $A X B C A$ and $A C B D A$ can contain no point in common. If at the same time $C$ were without $A X B D A$ it would follow that the interiors of $A C B D A$, $A X B D A$, and $A X B C A$ are mutually exclusive. But this would be contrary to Theorem 26. It is thus established that if $D$ is without $A X B C A$ then $C$ is within $A X B D A$. It follows by Theorem 25 that in this case the interior of $A X B D A=A C B+$ the interior of $A X B C A+$ the interior of $A C B D A$.

Theorem $\overline{28}$. If $O$ is a point on the closed curve $J$ and $\bar{R}$ is a region about $O$ then if $M$ denotes either the interior or the exterior of $J$, there exists a simple continuous arc $A X B$ such that (1) $A$ and $B$ are on $J$, (2) $A X B$ is common to $M$ and $\bar{R}$, (3) of the two arcs into which $A$ and $B$ divide $J$ that one which contains $O$ lies in $\bar{R}$.

Proof. By Axioms 6 and 7 there exist in $\bar{R}$ regions $L$ and $\bar{L}$ such that $L$ contains $O, \bar{L}$ lies in $M$ and all those points of $J$ which lie in $L$ belong to the boundary of $\bar{L}$. There exist on $J$ two points $A_{1}$ and $B_{1}$ such that the arc $A_{1} O B_{1}$ (on $J$ ) lies in $L$. Since $A_{1} O B_{1}$ is in $L$ and on $J$ it must be a part of the boundary of $\bar{L}$. There exist on $A_{1} O B_{1}$ points $A_{2}$ and $B_{2}$ in the order $A_{1} A_{2} O B_{2} B_{1}$. There exist regions $R_{A}$ and $R_{B}$ about $A_{2}$ and $B_{2}$ respectively such that $R_{A}$ contains no point of $A_{1} B_{1} O$ and $R_{B}$ contains no point of $B_{1} A_{1} O$, where $A_{1} B_{1} O$ and $B_{1} A_{1} O$ are intervals of $J$. There exist in $\bar{L}$ three distinct points $P, C$, and $D$ such that $C$ is in $R_{A}$ and $D$ is in $R_{B}$. There exist in $\bar{L}$ arcs $P C$ and $P D$. In $R_{A}$ and $R_{B}$ respectively there exist $\operatorname{arcs} C A_{2}$ and $D B_{2}$. The point-set $A_{2} C+C P+P D+D B_{2}$ contains as a subset an arc $A X B$ satisfying conditions (1), (2), and (3).

Theorem 29. If on the closed Jordan curve $J$ the points $A$ and $B$ separate the points $C$ and $D$ and $A X B$ and $C Y D$ are arcs such that $A X B$ and $C Y D$ are either both within or both without $J$ then $A X B$ and $C Y D$ have at least one point in common.

Proof. Case I. Suppose that $A X B$ and $C Y D$ are both within $J$. By Theorem 25, $R=R_{1}+R_{2}+A X \bar{B}$, where $\overline{R, R_{1}}$, and $R_{2}$ are the interiors of $J, A X B C A$, and $A X B D A$ respectively. There exists about $C$ a region $K$ containing no point of $R_{2}^{\prime}$. Since $C$ is a limit point of $C Y D, K$ contains a point $P$ belonging to $C Y D$. The point $P$ is in $R_{1}$ while $D$ is without $R_{1}$. Hence the interval $P \bar{D}$ of the arc $C Y D$ contains a point on the boundary of $R_{1}$. Every such point must be on $\underline{A X B}$. 
Case II. Suppose that $A X B$ and $C Y D$ are both without $J$. Then, by Theorem 27, either $C$ is without $A X B \overline{D A}$ or $D$ is without $A X B C A$. Suppose that $C$ is without $A X B D A$. Then $R_{1}=R+A D B+R_{2}$ where $R, R_{1}$, and $R_{2}$ are the interiors of $J, A X B C A$, and $A \overline{X B D} A$ respectively. The point $D$ is a limit point of $C Y D$. Hence $R_{1}$ contains a point $P$ belonging to $C Y D$. But $C Y D$ contains no point of $R^{\prime}$. Hence $P$ belongs to $R_{2}$. But $C$ is without $R_{2}$. Therefore the interval $C P$ of the arc $C Y D$ contains a point in common with the boundary of $R_{2}$. Every such point must be on $A X B$.

THEOREM 30. Under the same hypothesis as in Theorem 29, the interiors of $A X B D A$ and $C Y D B C$ have at least one point in common.

Theorem 31. If $C D$ is an arc, $B$ is a point of $C D$, and $B X$ is an arc which has no point except $B$ in common with $C D$ and $R$ is a region containing $B$ then there exists in the region $R$ an arc $E F G$ such that (1) the points $E$ and $G$ are on $C D$ in the order CEBGD, (2) the segment EBG of CD lies in $R$, (3) EFG has no point in common with $C D$, (4) there exists on $B X$ a point $H$ such that the segment $B H$ of $B X$ is (except for the point $B$ ) entirely within the closed curve EFGBE.

Proof. About $B$ (Fig. 3) there exists a region $\bar{R}$ which contains neither $C$ nor $D$. There exists an $\operatorname{arc} C Y D$ which lies entirely without $R$. There exist points $C_{1}$ and $D_{1}$ such that $C_{1}$ is the last point that $C Y D$ has in common with $C B$ and $D_{1}$ is the first point following $C_{1}$ that $C Y D$ has in common with $B D$. The arc $C_{1} X_{1} D_{1}$ (on $C Y D$ ) has no point except $C_{1}$ in common

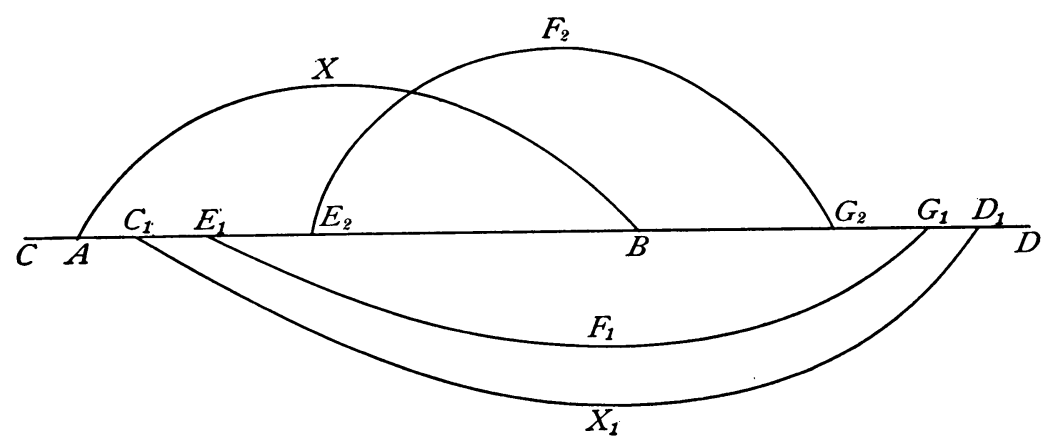

FIG. 3

with $C D$. About $B$ there is a region $K$ which contains no point of the closed point-set $C C_{1}+C_{1} X_{1} D_{1}+D_{1} D$. The regions $K$ and $R$ contain in common a region $R_{1}$ which contains $B$. By Theorem 28 there exists an arc $E_{1} F_{1} G_{1}$ such that (1) the points $E_{1}$ and $G_{1}$ are on $C D$ in the order $C_{1} E_{1} B G_{1} D_{1}$ (2) the closed curve $E_{1} F_{1} G_{1} B E_{1}$ is a subset of $R_{1}$, (3) $E_{1} F_{1} G_{1}$ is a subset of the interior of the closed curve $C_{1} X_{1} D_{1} B C_{1}$. About $B$ there is a region $H$ containing no point of the closed point-set $C E_{1}+E_{1} F_{1} G_{1}+G_{1} D$. The 
regions $H$ and $R$ contain in common a region $R_{2}$ that contains $B$. By Theorem 28 there exists an arc $E_{2} F_{2} G_{2}$ such that (1) the points $E_{2}$ and $G_{2}$ are on $C D$ in the order $C_{1} E_{1} E_{2} B G_{2} G_{1} D_{1}$, (2) the closed curve $E_{2} F_{2} G_{2} B E_{2}$ is a subset of $R_{2}$, (3) $E_{2} F_{2} G_{2}$ is a subset of the exterior of the closed curve $E_{1} F_{1} G_{1} B E_{1}$. The curve $E_{1} E_{2} F_{2} G_{2} G_{1} F_{1} E_{1}$ encloses a Jordan region $R_{3}$. By Theorem 25, $R_{3}=R_{1}+R_{2}+E_{2} B G_{2}$ where $R_{1}$ and $R_{2}$ are the interiors of $E_{1} F_{1} G_{1} B E_{1}$ and $E_{2} F_{2} G_{2} B E_{2}$ respectively. Since the connected pointset $B X A$ contains no point of $E_{2} B G_{2}$ therefore it cannot contain a point in $R_{1}$ and also a point in $R_{2}$. But since $B$ is a limit point of $B X$ the latter must contain points in either $R_{1}$ or $R_{2}$. It follows that there exists on $B X$ a point $H$ such that the point-set $B H-B$ is a subset of $R_{1}$ or a subset of $R_{2}$.

Theorem 32. If, in a domain $H, A B$ is an arc, $A_{1}, B_{1}$, and $D$ are ihree points on $A B$ in the order $A A_{1} D B_{1} B$ and $A_{1} X_{1} B_{1}$ is an arc which has no point in common with $A B$ except $A_{1}$ and $B_{1}$, and finally $C$ is a point in $R$, the interior of the closed curve $A_{1} X_{1} B_{1} D A_{1}$, then there exists, in $H$, an arc $C B$ which has no point except $B$ in common with $A B$.

Proof. There exists an infinite sequence of regions, $R_{1}, R_{2}, R_{3}, \cdots$ satisfying conditions (1), (2), and (3) of Theorem 5 except that $P$ is replaced by $B$. A point $P$ belonging to $A B$ is said to be in Class $\mathrm{I}$ it there exists, in $H$, a system of points $A_{2}, A_{3}, \cdots, A_{n}, B_{2}, B_{3}, \cdots, B_{n}$ on $A B$ and $\operatorname{arcs} A_{2} X_{2} B_{2}$, $\cdots, A_{n} X_{n} B_{n}$ such that

(1) $A_{i}$ and $B_{i}$ separate $A_{i+1}$ from $B_{i+1}$

$(1 \leqq i<n) ;$

(2) $P$ lies between $A_{n}$ and $B_{n}$;

(3) $A_{i} X_{i} B_{i}$ has no point in common with $A B$

$(1<i \leqq n)$;

(4) the interior of the closed curve $A_{i} X_{i} B_{i} B_{i-1} A_{i}(1<i \leqq n)$ does not contain either of the points $C$ and $B$;

(5) there exists on $B_{i} X_{i} A_{i}$ a point $H_{i}$ such that $B_{i} H_{i}-B_{i}$ lies in $R_{i+1}$, the interior of $A_{i+1} B_{i} B_{i+1} X_{i+1} A_{i+1}(1 \leqq i<n)$.

Under these conditions the arc $D P$ is said to be covered by the set of arcs $A_{1} X_{1} B_{1}, A_{2} X_{2} B_{2}, \cdots, A_{n} X_{n} B_{n}$.

Suppose there exist on $A B$ points that do not belong to Class I. Let Class II denote the set of all such points. Then there exists on $A B$ a point $O$ which, in the order from $A$ to $B$ on $A B$, is either the last point in Class I or the first point in Class II. About $O$ there is a region $\bar{R}$ that lies in $H$ and containing neither $C$ nor $B$ nor any point of the interval $A B_{1}$ of the arc $A B$. There exist* points $\bar{A}_{1}, \bar{A}_{2}, \bar{B}_{1}, \bar{B}_{2}$ on $A B$ in the order $A \bar{A}_{1} \bar{A}_{2} O \bar{B}_{2} \bar{B}_{1} B$ and $\operatorname{arcs} \bar{A}_{1} \bar{X}_{1} \bar{B}_{1}, \bar{A}_{2} \bar{X}_{2} \bar{B}_{2}$, having no point in common with $A B$ except their endpoints, such that the closed curves $\bar{A}_{1} \bar{X}_{1} \bar{B}_{1} O \bar{A}_{1}$ and $\bar{A}_{2} \bar{X}_{2} \bar{B}_{2} O \bar{A}_{2}$ lie in $\bar{R}$ and such that the interiors of these closed curves have no point in common. The arc $D \bar{A}_{2}$ can be covered by a finite set, $G$, of arcs. It follows $\dagger$

\footnotetext{
* Cf. proof of Theorem 31 .
}

$\dagger$ Cf. proof of Theorem 31 . 
that $D O$ may be covered by a finite set, $\bar{G}$, of arcs composed of a subset of $G$ together with either $\bar{A}_{1} \bar{X}_{1} \bar{B}_{1}$ or $\bar{A}_{2} \bar{X}_{2} \bar{B}_{2}$ and possibly* one additional arc. But if $\bar{G}$ covers $D O$ it must also cover $D P_{2}$ where $P_{2}$ is some point that follows $O$ and therefore belongs to Class II. Thus the supposition that Class II exists leads to a contradiction. Hence if $P$ is any point of $A B, D P$ can be covered by a finite set of arcs. It easily follows that there exists an infinite set of arcs $A_{2} X_{2} B_{2}, A_{3} X_{3} B_{3}, \cdots$ which satisfy conditions (1), (2), (3), (4), and (5) ( $\infty$ being substituted for $n$ ) and are such that, if $m$ is a positive integer, there exists $\delta$ such that if $n>\delta$ then $A_{n} X_{n} B_{n} B_{n-1} A_{n}$ is a subset of $R_{m}$. Let $\bar{R}_{i}$ denote the interior of the closed curve $A_{i} X_{i} B_{i} B_{i-1} A_{i}$. For every $i, \bar{R}_{i}$ has a point in common with $\bar{R}_{i+1}$. By Zermelo's postulate there exists a sequence of points $P_{1}, P_{2}, P_{3} \cdots$ such that $P_{i}$ is common to $\bar{R}_{i}$ and $\bar{R}_{i+1}$. There exists in $\bar{R}_{1}$ an arc $C P_{1}$ and in $\bar{R}_{i}(i>1)$ an arc $P_{i-1} P_{i}$. There exists a point $\bar{P}_{2}$ which is the first point that $C P_{1}$ has in common with $P_{1} P_{2}$. The arc $C \bar{P}_{2}$ (on $C P_{1}$ ) and the $\operatorname{arc} \bar{P}_{2} P_{2}$ (on $P_{1} P_{2}$ ) constitute together an arc $C P_{2}$. Similarly there exists an arc $C P_{3}$ formed by the arc $C \bar{P}_{3}$ (on $C P_{2}$ ) together with the arc $\bar{P}_{3} P_{3}$ (on $\left.P_{2} P_{3}\right)$ where $\bar{P}_{\mathrm{s}}$ is the first point that $C P_{2}$ has in common with $P_{2} P_{3}$. In general $C P_{i+1}$ denotes $C \bar{P}_{i+1}+\bar{P}_{i+1} P_{i+1}$ where $\bar{P}_{i+1}$ is the first point that $C P_{i}$ has in common with $P_{i} P_{i+1}$. For a given $\bar{n}$ there exists $n_{\bar{n}}$ such that if $n>n_{n}^{-}$then $C P_{n}$ contains $C P_{\bar{n}}$. It follows that for a given $n$ there exists an arc $C X_{n}$ which is common to all the arcs $C P_{n}$, $C P_{n+1}, C P_{n+2}, \cdots$, and contains every other arc which is common to ti.e arcs of this sequence and has $C$ for one end point. For every $m, C X_{m+1}$ contains $C X_{m}$ and there exists $r$ such that $C X_{m}$ is a proper subset of $C X_{m+r}$. Let $\tau$ denote the point-set $B+C X_{1}+C X_{2}+\cdots$. It will be shown that $\tau$ satisfies Lennes' definition of a Jordan arc from $C$ to $B$.

First $\tau$ is closed. For suppose that $P$ is a limit point of $\tau$ that does not belong to $\tau$. Then clearly $P$ is not a limit point of any single $C X_{n}$. But if $R$ is a region about $B$ then there exist $m$ and $\delta$ such that (1) $R_{m}^{\prime}$ is a subset of $R$, (2) for every $n$ greater than $\delta, \tau-C X_{n}$ is a subset of $R_{m}^{\prime}$. But $P$ is a limit point of $\tau-C X_{n}$. Hence $P$ is in $R$. It follows that $P$ is identical with $B$. Thus the supposition that $P$ does not belong to $\tau$ leads to a contradiction. It follows that $\tau$ is closed. It is easily seen that $B$ is a limit point of $C X_{1}+C X_{2}+\cdots$ and that $\tau$ is connected and contains no proper connected subset that contains $B$ and $C$. Thus $\tau$ is a simple continuous arc from $C$ to $B$. Clearly it contains no point of $A B$ except $B$.

Theorem 33. If $A$ is a point on the boundary of a region $R, B$ is a point in $R$, and $A B$ is an arc such that $A B$ is in $R$, then $R-A B-A)$ is connected. Proof (on the basis of Axioms 2-5, 8 and Theorems 5, 15, and 32) for the

* An additional arc may be necessary in case $\bar{A}_{1}$ or $\bar{A}_{2}$ coincides with an endpoint of some arc of the set $G$. 
case where the boundary of $R$ is a closed curve $J$. On $J$ (Fig. 4) there is a point $O$ different from $A$. About $O$ there is a region $\bar{R}$ which contains no point of $A B$. The region $\bar{R}$ contains a point $C$ in common with $R$. There exists an arc $B C$ such that $B C$ is in $R$ and an arc $C O$ lying in $\bar{R}$. The point-set $B C+C O$ contains as a subset an $\operatorname{arc} D E$ where $D$ is a point of $A B, E$ is a point in $\bar{R}$ and on $J$ and $D E$ is a subset of $R-(A B-A)$. The arc $A D E$ divides $R$ into two regions of which one contains $D B-D$ and the other one contains no point of $A B$. Let $L$ denote the latter region and let $F$ denote a point in $L$. By Theorem 32 there exists an arc $F B$

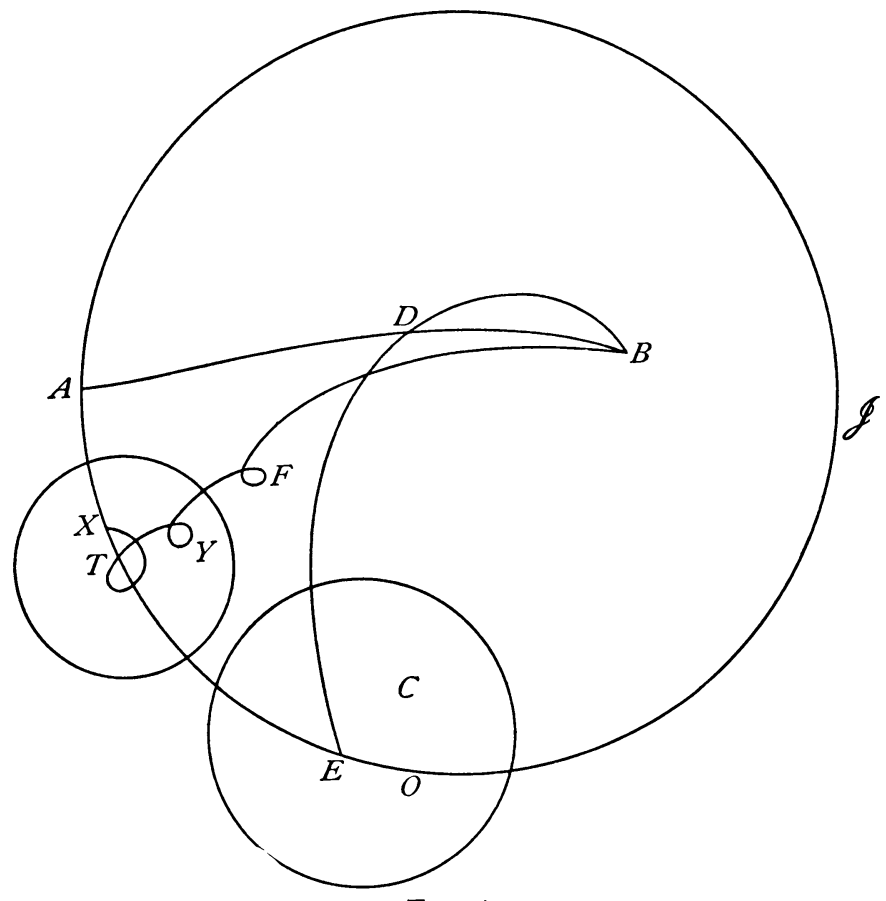

FIG. 4

which has no point in common with $A B$. Let $A X E$ denote that arc from $A$ to $E$ on $J$ which forms a part of the boundary of $L$. About $X$ there is a region $K$ which contains no point of $A D+D E$. The regions $K$ and $L$ have a point $Y$ in common. There exists in $L$ an arc $F Y$ and in $K$ an arc $Y X$. The point-set $B F+F Y+Y X$ contains as a subset an arc $B T$ where $T$ is a point on $J$ and $B T$ is a subset of $R-(A B-A)$. The points $A$ and $T$ divide $J$ into two $\overline{\operatorname{arcs}} A M T$ and $A N T$. By Theorem 25, $R=R_{1}+R_{2}+A B+B T$, where $R_{1}$ and $R_{2}$ are the interiors of $A B T M A$ and $A B T N A$ respectively. Suppose now that $P_{1}$ and $P_{2}$ are two points in $R-(A B-A)$. If $P_{1}$ and $P_{2}$ are both in $R_{1}$ or both in $R_{2}$ there exists 
an $\operatorname{arc} P_{1} P_{2}$ lying entirely in $R_{1}$ or entirely in $R_{2}$. Suppose $P_{1}$ is in $R_{1}$ and $P_{2}$ is in $R_{2}$. There exists on $B T$ a point $Z$. About $Z$ there is a region $R_{3}$ containing no point of $A B$. There exist arcs $P_{1} Z_{1}$ and $P_{2} Z_{2}$ such that $P_{1} Z_{1}$ is in $R_{1}, P_{2} Z_{2}$ is in $R_{2}$ and the points $Z_{1}$ and $Z_{2}$ are in $R_{3}$ and on the boundaries of $R_{1}$ and $R_{2}$ respectively and therefore on $B T$. If $Z_{1} Z_{2}$ denotes that arc from $Z_{1}$ to $Z_{2}$ which is a subset of $B T$ then $\overline{P_{1}} Z_{1}+Z_{1} Z_{2}+Z_{2} P_{2}$ is an arc from $P_{1}$ to $P_{2}$ lying in $R-(A B-A)$. The case where one or both of the points $P_{1}$ and $P_{2}$ is on $D E$ can be easily treated. It follows that every two points in $R-(A B-A)$ can be joined by an arc lying entirely in $R-(A B-A)$. Hence $R-(A B-A)$ is connected.

Theorem 34. If $J$ is a Jordan arc and $A B$ is an arc lying wholly in $R$, the interior of $J$, then $R-A B$ is connected.

Proof (on the basis of Axioms 2-5, 8 and Theorems 5, 15, and 32) for the case where the boundary of $R$ is a closed curve $J$. There exists an $\operatorname{arc} A_{1} X_{1} B_{1}$ such that $A_{1}$ and $B_{1}$ are on $A B$ in the order $A A_{1} B_{1} B$ but $A_{1} X_{1} B_{1}$ has no point in common with $A B$. There exists a point $X$ in the interior of the closed curve formed by the arc $A_{1} X_{1} B_{1}$ and the interval $A_{1} O B_{1}$ of the arc $A B$. By Theorem 32 there exist $\operatorname{arcs} X A$ and $X B$ such that neither $X A$ nor $X B$ has a point in common with $A B$. The point-set $X A+X B$ contains as a subset an $\operatorname{arc} A Y B$. About $Y$ there is a region $\bar{R}$ which contains no point of $A B$. On $J$ there is a point $P$. There exists an arc $P E$ such that $P E$ is without $A Y B O A$ and such that $E$ is on $A Y B O A$ and in $\bar{R}$ (and therefore not on $A B)$. The arc $P E$, the arc from $E$ to $A$ on $A Y B$ and the arc $A B$ constitute together an arc $P E A B$. By Theorem $33, R-(P E A B-P)$ is connected. It easily follows that $R-A B$ is connected.

Proof of Theorems 33 and 34 (on the basis of Axioms 2-5, 8 and Theorems $5,15,32$, and 31 ) for the case where $R$ is any region.* Suppose $C$ and $D$ are two distinct points in $R$ but not on the arc $A B$. There exists, in $R$, an arc $C A$. Let $X$ denote the first point that $C A$ has in common with $A B$. Suppose $X$ is distinct from $A$. By Theorems 31 and 32, some point $F$ on $X A$ can be joined to $A$ by an arc $A F$ such that $A F-A$ is a subset of $(R+B)-A B$. It follows that there exists, in $R$, an arc $C A$ that has no point except $A$ in common with $A B$. Likewise there exists in $R$ an arc $D C$ having no point except $C$ in common with the arc $C A B$.

\section{Consequences of Axioms 2-5, 8 and Theorems 5, 15, 20, 33, And 34}

TheOREM 35. Every region contains at least one simple closed curve.

Proof. Suppose $R$ is a region. By Theorems 4 and 16, there exists in $R$ a simple continuous arc $A B$. On $A B$ there is a point $X$ distinct from $A$ and

\footnotetext{
* This proof is a modification of a proof due to Mr. J. R. Kline, one of my students.
} 
from $B$. By Theorem 23, $R$ contains a point $C$ which is not on $A B$. By Theorems 34 and 15 there is, in $R$, a simple continuous arc $A C$ which has no point in common with $X B$. Let $O$ denote the last point that $A B$ has in common with $A C$. There exists an arc $X O C$ consisting of $X O$ (on $B A$ ) together with $O C$ (on $A C$ ). On $O C$ there is a point $T$. By Theorem 34, there is in $R$ an arc $B C$ which has no point in common with $X O T$. There exist $E$ and $F$ such that $E$ is the last point that $B C$ has in common with $B A$ and $F$ is the first point that $O C$ has in common with $E C$. Clearly $E O$ (on $B A$ ) $+O F$ (on $O C$ ) $+F E$ (on $C B$ ) is a closed curve lying wholly in $R$.

Theonem 36. If $O$ is a point in a region $R$ there exists a simple closed curve which lies in $R$ and encloses $O$.

Proof. By Theorem 4 there exists in $R$ a point $X$ distinct from $O$. By Axiom 1 there exists about $X$ a region which lies in $R$ and does not contain $O$. By Theorem 35 this region contains a closed curve $J$ (Fig. 5). By Theorem 21, $O$ is without $J$. On $J$ there is a point $A_{1}$. In $R$ there is an $\operatorname{arc} A_{1} O$. There exists a point $A$ which is the last point that $A_{1} O$ has in common with $J$. The

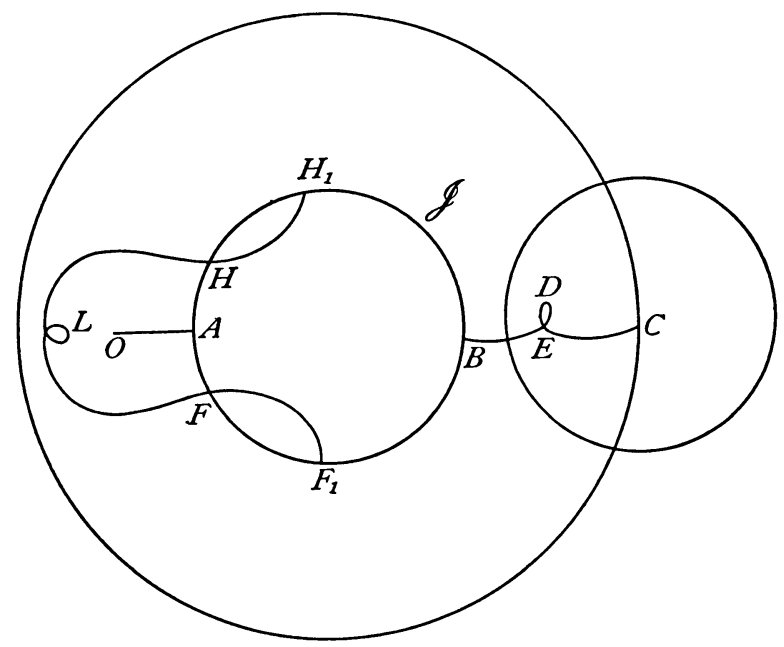

FIG. 5

$\operatorname{arc} A O$ has no point except $A$ in common with $J$. By Theorem 19 there exists a point $C$ on the boundary of $R$. There exists about $C$ a region $\bar{R}$ containing no point of the closed point-set $O A+J+I$, where $I$ is the interior of $J$. The region $\bar{R}$ contains at least one point $D$ of $R$. On $J$ there is a point $B_{1}$ distinct from $A$. By Theorem 34 there exists, in $R-A O$, an arc $B_{1} D$. Let $B$ denote the last point that $B_{1} D$ has in common with $J$. There exists in $\bar{R}$ an arc $D C$. The arc $D C$ can contain no point of $O A+J+I$. Let $E$ be the last point which $D C$ has in common with $B D$. Then $B E$ (on $B D)+E C$ (on $D C$ ) is 
an $\operatorname{arc} B C$ every point of which, except $C$, is in $R$ and no point of which, except $B$, belongs to $O A+J+I$. The points $A$ and $B$ divide the curve $J$ into two arcs $A F_{1} B$ and $A H_{1} B$. There exists, about $F_{1}$, a region $R_{1}$ which contains no point of the closed point-set $O A+A H_{1} B+B C+(S-R)$. By Theorem 20, $R_{1}$ contains a point $L$ not in $J+I$. There exists in $R$ an arc $L F_{1}$. There exists a point $F$ which is the first point that $L F_{1}$ has in common with $J$. By Theorem 33 there is, in $R$, an arc $L H_{1}$ which has no point in common with $O A+A F_{1} B+B C$. There is a point $H$ which is the first point that $L H_{1}$ has in common with $J$. The point-set $L F$ (on $L F_{1}$ ) $+L H$ (on $L H_{1}$ ) contains as a subset an arc $F Z H$ which, except for its endpoints, is a subset of $R-(J+I)$. By Theorem 21 the interior of the closed curve $Z H A F Z$ is in $R$. Hence $C$ is without $Z H A F Z$. Therefore if $B$ were within $Z H A F Z, B C$ would have a point in common with $Z H A F Z$ and therefore a point in common with $L F_{1}$ or $L H_{1}$ or $J$. It follows that $B$ is without $Z H A F Z$. Hence, by Theorem $27, A$ is within the closed curve $Z H B F Z$. But the arc $O A$ contains no point of $Z H B F Z$. Hence $O$ is within $Z H B F Z$.

Theorem 37. If $J$ and $C$ are simple closed curves, $O$ is a point on $J$ but not on $C, A_{1}$ and $A_{2}$ are distinct points common to $C$ and $J$ and $A_{1} X A_{2}$ is an arc on $C$ such that $A_{1} X A_{2}$ lies within $J$ then there exist two points $O_{1}$ and $O_{2}$ distinct from $O$ such that

(1) $O_{1}$ and $O_{2}$ lie on the arcs* $A_{1} O$ and $A_{2} O$ respectively,

(2) there is on the curve $C$ an arc $\mathrm{O}_{1} \mathrm{YO}_{2}$ such that $\mathrm{O}_{1} \mathrm{YO}_{2}$ is within $J$,

(3) if $B_{1}$ and $B_{2}$ are points on the arcs* $O_{1} O$ and $\bar{O}_{2} O$ respectively such that there exists on $C$, from $B_{1}$ to $B_{2}$, an arc which, except for its endpoints, lies entirely within $J$, then $B_{1}=O_{1}$ and $B_{2}=O_{2}$.

Proof. Let $M_{1}$ be the set of all points [ $\left.P_{1}\right]$ on $A_{1} O$ such that $P_{1}$ can be joined to some point of $A_{2} O$ by an arc of $C$ which, except for its endpoints, lies within $J$. Let $M_{2}$ be the set of all points [ $P_{2}$ ] on $A_{2} O$ such that $P_{2}$ can be joined to some point $X$ of $M_{1}$ by an arc of $C$ which, except for its endpoints, lies within $J$, but such that no point between $P_{2}$ and $O$ on $A_{2} O$ can be so joined to the same point $X$. Suppose that $Z_{1}$ and $W_{1}$ are two distinct points of $M_{1}, Z_{2}$ and $W_{2}$ are points of $M_{2}$ and $Z_{1} Z_{2}$ and $W_{1} W_{2}$ are arcs such that $Z_{1} Z_{2}$ and $W_{1} W_{2}$ are within $J$. The arcs $Z_{1} Z_{2}$ and $W_{1} W_{2}$ can have no point in common. $\dagger$ Hence, in view of Theorem 29, it is clear that if $W_{1}$ is between $Z_{1}$ and $O$ on the arc $A_{1} O$ then $W_{2}$ must be between $Z_{2}$ and $O$ on the $\operatorname{arc} A_{2} O$.

If Theorem 37 is false there exists a point $L_{1}$ on $A_{1} O$ and a point $L_{2}$ on $A_{2} O$ such that $L_{1}$ is a limit point of $M_{1}, L_{2}$ is a limit point of $M_{2}$, but $L_{1} O L_{2}$

* Here $A_{1} O, A_{2} O, O_{1} O$, and $O_{2} O$ denote intervals of the arc $A_{1} O A_{2}$ on $J$.

$\dagger$ Two arcs of a closed curve can have no point in common unless one of them contains an endpoint of the other one. 
contains no point either of $M_{1}$ or of $M_{2}$. On the curve $C$ there exists a point $E$ such that, on $C, A_{1}$ and $L_{2}$ separate $L_{1}$ from $E$. On the $C$-arc $A_{1} L_{1} L_{2} E$ there exist points $F$ and $G$ in the order $L_{1} F G L_{2}$. Since $L_{1}$ and $L_{2}$ are limit points of $M_{1}$ and $M_{2}$ respectively therefore $M_{1}$ contains two distinct points $P_{1}$ and $\bar{P}_{1}$ and $M_{2}$ contains two distinct points $P_{2}$ and $\bar{P}_{2}$ such that (1) on the arc $A_{1} L_{1} L_{2} E, P_{1}$ and $\bar{P}_{1}$ lie between $A_{1}$ and $F$ while $P_{2}$ and $\bar{P}_{2}$ lie between $E$ and $G$, (2) there exist, on $C$, arcs $P_{1} P_{2}$ and $\bar{P}_{1} \bar{P}_{2}$ such that $P_{1} P_{2}$ and $\bar{P}_{1} \bar{P}_{2}$ lie entirely within $J$.

Since $A_{1}$ is on $J$, neither $P_{1} P_{2}$ nor $\bar{P}_{1} \bar{P}_{2}$ can contain $A_{1}$. Hence both of these arcs contain $F$ and $G$. But it has been previously established that $P_{1} P_{2}$ and $\bar{P}_{1} \bar{P}_{2}$ have no point in common. Thus the supposition that Theorem 37 is false leads to a contradiction.

Theorem 38. If $J$ and $C$ are closed curves, $O$ is a point on $J$ but not on $C$, $A_{1}$ and $A_{2}$ are distinct points common to $C$ and $J$, and $A_{1} X A_{2}$ is an arc on $C$ such that the interior of the closed curve $A_{1} X A_{2} O A_{1}$ is a subset of the exterior of $J$ then there exist two points $O_{1}$ and $O_{2}$, distinct from $O$, such that (1), on the interval $O_{1} O O_{2}$ of the curve $J, O$ is between $O_{1}$ and $A_{2}$ and between $O_{2}$ and $A_{1}$, (2) there is on the curve $C$ an arc $O_{1} Y O_{2}$ such that $O_{1} Y O_{2}$ and the interior of the closed curve $\mathrm{O}_{1} \mathrm{YO}_{2} \mathrm{OO}_{1}$ are subsets of the exterior of $J,(3)$ if the exterior of $J$ contains $B_{1} Z B_{2}$ and the interior of the closed curve $B_{1} Z B_{2} O B_{1}$ where $B_{1}$ and $B_{2}$ are points on that arc of $J$ from $O_{1}$ to $O_{2}$ which does not contain $O$, and $B_{1} Z B_{2}$ is an arc of $C$, then $B_{1}=O_{1}$ and $B_{2}=O_{2}$.

Theorem 39. If $O$ is a point on a closed curve $J$ then every point not on $J$ can be joined to $O$ by an arc having no point except $O$ in common with $J$.

Proof. It may be easily shown with the help of Theorems 5 and 36 that there exists a sequence of closed Jordan curves $J_{1}, J_{2}, \cdots$, such that (1) the interior of $J_{1}$ does not contain $I$, the interior of $J$, (2) for each $n, J_{n+1}$ lies in $I_{n}$, the interior of $J_{n}$, (3) $O$ is the only point that the regions $I_{1}, I_{2}, \cdots$ have in common, (4) it $Q$ is a region about $O$ there exists $n$ such that $I_{n}^{\prime}$ lies in $Q$.

By Theorem 20, $I_{n}$ contains at least one point of $E$, the exterior of $J$. Hence, by Theorem $18, E=E_{n}+\bar{E}_{n}$ where $E_{n}$ is a subset of $I_{n}$ but no point of $\bar{E}_{n}$ belongs to $I_{n}$. By Axiom $3, \bar{E}_{n}$ must contain a point $P$ which is a limit point of $E_{n}$. Every such point $P$ must belong to $J_{n}$. With the use of (1) it may be shown that $I$ contains a point of $J_{n}$. Thus $J_{n}$ contains a point within $J$ as well as a point without $J$ and therefore it must contain at least two points in common with $J$.

It follows that there exist, on $J$, two points $A$ and $B$ (Fig. 6), belonging to $J_{n}$, such that no point of $J_{n}$, except $A$ and $B$, is on the $\operatorname{arc} A O B$. If $X$ is a point of $J_{n}$ that lies in $I$ then $J_{n}$ and $J$ have in common two points $\bar{A}_{x}$ and $\bar{B}_{x}$ such that the $J_{n}$-arc $\bar{A}_{x} X \bar{B}_{x}$ lies (except for its endpoints) in $I$. By Theorem 37 there exists a $J_{n}$-arc $A_{x} B_{x}$ such that 
(1) $A_{x}$ is $\bar{A}_{x}$ or is on $J$ in the order $\bar{B}_{x} \bar{A}_{x} A_{x} O$,

(2) $B_{x}$ is $\bar{B}_{x}$ or is on $J$ in the order $\bar{A}_{x} \bar{B}_{x} B_{x} O$,

(3) $A_{x} B_{x}$ is in $I$,

(4) if $Y$ and $Z$ are points on $J$ on the arcs* $A_{x} O$ and $B_{x} O$ respectively such that an arc of $J_{n}$ from $Y$ to $Z$ lies (except for its endpoints) in $I$, then $Y=A_{x}$ and $Z=B_{x}$. It is clear that, for a given $n$, the $\operatorname{arc} A_{x} B_{x}$ is completely determined by the point $X$. For every $X$ on $J_{n}$ that lies in $I$ construct the corresponding $A_{x} B_{x}$. Let $\bar{J}_{n}$ denote the point-set which is composed of all

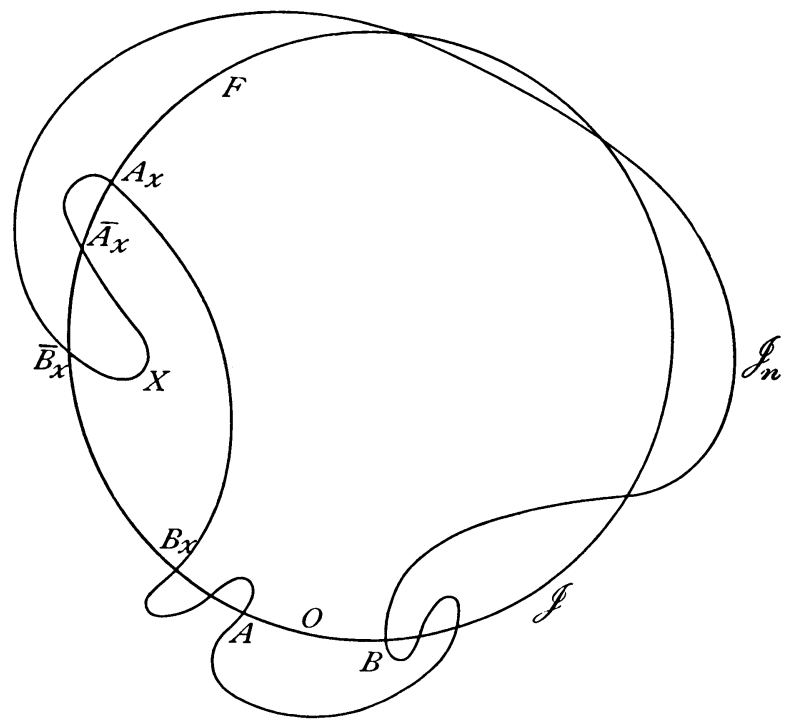

FIG. 6

the $A_{x} B_{x}$ 's so constructed together with every point $F$ on $J$ which has the property that for no $X$ is $F$ separated from $O$ by $A_{x}$ and $B_{x}$. It may be easily proved that $\bar{J}_{n}$ is a closed Jordan curve. By Theorem $21, \bar{I}_{n}$ (the interior of $\left.\bar{J}_{n}\right)$ is a subset of $I$. That $\bar{I}_{n}$ is a subset of $I_{n}$ may be proved as follows.

Let $F$ be a point of $\bar{J}_{n}$ that does not belong to $J_{n}$. The curves $J_{n}$ and $J$ have in common two points $C$ and $D$ such that the $J$-arc $C F D$ contains no point of $J_{n}$. About $F$ and $O$ respectively there exist regions $R_{1}$ and $R_{2}$ neither of which contains a point of the closed point-set $J_{n}+(J-A O B-C F D)$. Since $F$ and $O$ are on its boundary the region $\bar{I}_{n}$ contains a point $P_{1}$ in $R_{1}$ and a point $P_{2}$ in $R_{2}$. There exists an arc $P_{1} P_{2}$ lying entirely in $\bar{I}_{n}$ and therefore having no point in common with $J_{n}$. It easily follows $\dagger$ that there exists an $\operatorname{arc} T_{1} T_{2}$ such that (1) $T_{1}$ is on $J$ and in $R_{1}$ and therefore on $C F D$, (2) $T_{2}$ is on $J$ and in $R_{2}$ and therefore on $A O B$, (3) $T_{1} T_{2}$ is in $\bar{I}_{n}$. But neither

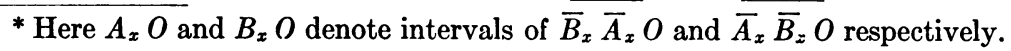

†Cf. proof of Theorem 25. 
$A O B$ nor $C F D$ contains a point of $J_{n}$. It follows that $F$ can be joined to $O$ by an arc $F T_{1} \dagger+T_{1} T_{2}+T_{2} O^{*}$ that has no point in common with $J_{n}$. But $O$ is within $J_{n}$. Hence $F$ also is within $J_{n}$. Thus every point of $\bar{J}_{n}$ is either on or within $J_{n}$. Hence $I_{n}$ is a subset of $I_{n}$.

For every $n, \bar{I}_{n+1}$ is a proper subset of $\bar{I}_{n}$. In $I_{1}$ there is a point $P_{1}$ not belonging to $\bar{I}_{2}$. In $\bar{I}_{2}$ there is a point $P_{2}$. There exists an $\operatorname{arc} P_{1} P_{2}$ lying in $\bar{I}_{1}$. Since the regions $\bar{I}_{1}, \bar{I}_{2}, \ldots$ have no point in common there exists $n_{2}$ such that $P_{1} P_{2}$ contains a point $P_{n_{2}}$ lying in $I_{n_{2}}$ but contains no point in $I_{n_{2}+1}$. In $I_{n_{2}+1}$ there is a point $P_{n_{2}+1}$. There exists an arc $P_{n_{2}} P_{n_{2}+1}$ lying in $I_{n_{2}}$. Let $\bar{P}_{n_{2}}$ denote the first point that $P_{1} P_{2}$ has in common with $P_{n_{2}} P_{n_{2}+1}$. Similarly there exists $n_{3}$ such that $P_{n_{2}} P_{n_{2}+1}$ contains a point $P_{n_{3}}$ in $I_{n_{3}}$ but no point in $I_{n_{3}+1}$. There exists in $I_{n_{3}+1}$ a point $P_{n_{3}+1}$ and in $I_{n_{3}}$ an are $P_{n_{3}} P_{n_{3}+1}$. Let $\bar{P}_{n_{3}}$ denote the first point that $P_{n_{9}} P_{n_{2}+1}$ has in common with $P_{n_{3}} P_{n_{3}+1}$. Continue this process. In general, if $k>1$, there exists $n_{k+1}$ such that $P_{n_{k}} P_{n_{k}+1}$ contains a point $P_{n_{k+1}}$ in $I_{n_{k+1}}$ but no point in $I_{n_{k+1}+1}$. There exists in $I_{n_{k+1}+1}$ a point $P_{n_{k+1}+1}$ and in $I_{n_{k+1}}$ an arc $P_{n_{k+1}} P_{n_{k+1}+1}$. The first point that $P_{n_{k}} P_{n_{k}+1}$ has in common with $P_{n_{k+1}} P_{n_{k+1}+1}$ is denoted by $\bar{P}_{n_{k+1}}$. It mav be easily proved that the point-set $O+P_{1} \bar{P}_{n_{2}}$ (on $P_{1} P_{2}$ ) $+\bar{P}_{n_{2}} \bar{P}_{n_{3}}$ (on $\left.P_{n_{2}} P_{n_{2}+1}\right)+\bar{P}_{n_{3}} \bar{P}_{n_{4}}$ (on $\left.P_{n_{3}} P_{n_{3}+1}\right)+\cdots$ is an arc from $P_{1}$ to $O$. This arc $P_{1} O$ lies in $I$ and has no point except $O$ in common with $J$. If $P$ is any point in $I$ other than $P_{1}$ there exists an arc $P P_{1}$ lying in $I$ and the point-set $P P_{1}+P_{1} O$ contains as a subset an arc $P O$ which has no point except $O$ in common with $J$.

That a point without $J$ can be so joined to any point of $J$ may be proved in a similar manner.

Theorem 40. If $A$ and $B$ are two points on a Jordan curve $J, A$ and $B$ can be joined by Jordan arcs $A X B$ and $A Y B$ such that $A X B$ is within $J$ and $A Y B$ is without $J$.

Theorem 41. If $J_{1}$ and $J_{2}$ are two closed Jordan curves whose interiors $I_{1}$ and $I_{2}$ have a point $O$ in common then there exists a Jordan curve $J$, every point of which belongs to either $J_{1}$ or $J_{2}$, such that $I_{1}+I_{2}$ is a subset of $I$, the interior of $J$.

Proof. Case I. Suppose $J_{1}$ and $J_{2}$ have not more than one point in common. Then, since $I_{1}$ and $I_{2}$ have a point in common, it easily follows that either $I_{1}$ is in $I_{2}$ or $I_{2}$ is in $I_{1}$. In the former case $J$ is $J_{2}$. In the latter case it is $J_{1}$.

Case II. Suppose $J_{1}$ and $J_{2}$ (Fig. 7) have at least two points in common. If every point of $J_{1}$ is within or on $J_{2}$ then $J$ is $J_{2}$. Suppose that at least one point $P$ of $J_{1}$ is without $J_{2}$ and at least one point of $J_{2}$ is without $J_{1}$. Then there exist two points $\bar{A}$ and $\bar{B}$ common to $J_{1}$ and $J_{2}$ such that the $\operatorname{arc} \overline{A P} \bar{B}$

* Here $F T_{1}$ and $T_{2} O$ denote intervals of $C F D$ and $A O B$ respectively. 
of $J_{1}$ lies (except for its endpoints) entirely without $J_{2}$. The points $\bar{A}$ and $\bar{B}$ divide $J_{2}$ into two arcs. By Theorem 27 , one of these $\operatorname{arcs}, \bar{A} C \bar{B}$, is such that the curve $P \bar{B} C \bar{A}$ contains $I_{2}$. Let $\bar{A} D \bar{B}$ denote the other arc of $J_{2}$ from $\bar{A}$ to $\bar{B}$. By Theorem 38 there exist, on the arc $\bar{B} C \bar{A}$, points $A_{P}$ and $B_{P}$ such that $\bar{A}$ and $A_{P}$ do not separate $\bar{B}$ from $B_{P}$ and such that (1) there exists

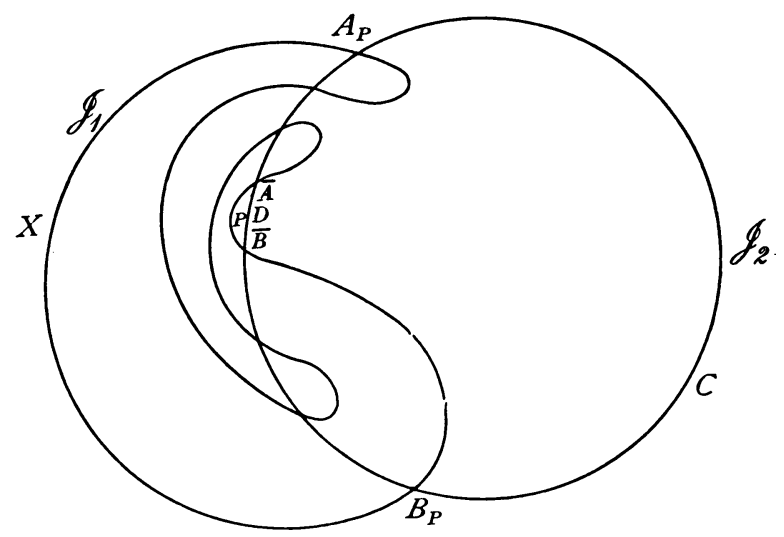

Fig. 7

an interval $A_{P} X B_{P}$ of $J_{1}$ lying entirely without $J_{2}$ except for its endpoints and such that if $\bar{C}$ is a point on $J_{2}$ that is separated from $D$ by $A_{P}$ and $B_{P}$ then $A_{P} X B_{P} \bar{C} A_{P}$ includes $I_{2}$ within it, (2) if $Z$ and $W$ are points on $A_{P} \bar{C}$ and $B_{P} \bar{C}$ respectively and there is, on $J_{1}$, an arc $Z V W$ lying entirely without $J_{2}$ except for its endpoints and such that $Z V W C Z$ includes $I_{2}$ within it then $Z=A_{P}$ and $W=B_{P}$. The curve $A_{P} X B_{P} \bar{C} A_{P}$ contains within it or on it every point of the curve $P \bar{A} A_{P} \bar{C} B_{P} \bar{B} P$ and every point of the curve $J_{2}$. For every point $P$ which is on $J_{1}$ but without $J_{2}$ construct the corresponding arc $A_{P} X B_{P}$. Call this arc $t_{P}$ and let $k_{P}$ denote that interval of $J_{2}$ from $A_{P}$ to $B_{P}$ which has the property that the interior of the closed curve $t_{P}+k_{P}$ is entirely without $J_{2}$. Let $J$ denote the point-set composed of all such arcs $t_{P}$ together with the set of all points [ $Y$ ] on $J_{2}$ such that for no $P$ is $Y$ on the arc $k_{P}$. It may be proved that $J$ is a closed curve satisfying the requirements of Theorem 41.

ThEOREM 42. If the sum of the interiors of a finite set $G$ of closed curves is a connected point-set then there exists a closed curve $J$ such that (1) every point of $J$ belongs to some curve of the set $G$, (2) the interior of $J$ contains the interiors of all the curves of the set $G$.

THEOREM 43. If the point $O$ of the closed curve $J$ is within every curve of the finite set of closed curves $G$ and the interior of $J$ is not a subset of the interior of any curve of the set $G$ then there exist curves $J_{1}$ and $J_{2}$ containing $O$ such that (1) every point on $J_{1}$ or on $J_{2}$ belongs to $J$ or to some curve of the set $G,(2)$ every 
point within $J_{1}$ is without $J$ while every point within $J_{2}$ is within $J$, (3) every point within $J_{1}$ or within $J_{2}$ is within every curve of the set $G$.

TheOREM 44. If the point $O$ of the closed curve $J$ is without every curve of the finite set of closed curves $G$ and every curve of $G$ has at least one point in common with the $\left\{\begin{array}{l}\text { interior } \\ \text { exterior }\end{array}\right\}$ of $J$ then there exists a closed curve $\bar{J}$ containing $O$ and such that (1) every point of $\bar{J}$ belongs either to $J$ or to some curve of the set $G$, (2) every point $\left\{\begin{array}{l}\text { without } \\ \text { within }\end{array}\right\} \bar{J}$ is $\left\{\begin{array}{l}\text { without } \\ \text { within }\end{array}\right\} J$ and without every curve of the set $G$.

Theorem 45. If $O$ is a point on a closed curve $J$ and $\bar{R}$ is a region containing $O$ then there exists an arc $A X B$ such that (1) $A$ and $B$ are on $J$, (2) the segment $A O B$ of the curve $J$ is in $\bar{R}$, (3) $A X B$ and the interior of $A O B X A$ are in $\bar{R}$ and without $J$.

Proof. On $J$ there is a point $P$ different from $O$. There exists about $O$ and within $\bar{R}$ a region $\bar{K}$ that does not contain $P$. By Theorem 36, there is in $\bar{K}$ a closed curve $\bar{J}$ enclosing $O$. There exist on $J$ two points $A$ and $B$ such that the segment $A O B$ of the curve $J$ is within $\bar{J}$. By Theorem 40 there exists an arc $A Y B$ such that $A Y B$ is without $J$. It easily follows with the aid of Theorem 27 that either the interior or the exterior of $A Y B O A$ is a subset of the exterior of $J$. Hence by Theorems 21 and 43 there exists an arc $A X B$ such that $A X B$ and the interior of $A X B O A$ are in $\bar{R}$ and without $J$.

TheOREM 46. If the closed curve $J$ has no point in common with the closed set of points $K$ then there exist closed curves $J_{1}$ and $J_{2}$ such that $J$ lies between* $J_{1}$ and $J_{2}$ but no point of $K$ lies between $J_{1}$ and $J_{2}$.

Proof. If $P$ is a point of $J$ there exists, about $P$, a region $\bar{R}$ containing no point of the closed point-set $K$. By Theorem 45 there exist two points $A$ and $B$ and an arc $A X B$ such that (1) $A$ and $B$ are on $J$, (2) the segment $A P B$ of the curve $J$ is in $\bar{R}$, (3) $A X B$ and the interior of $A P B X A$ are in $\bar{R}$ but without $J$. It easily follows with the aid of the Heine-Borel Theorem (as applied to a set of segments covering a closed curve) that there exist a finite set of $J-\operatorname{arcs} A_{1} B_{1}, A_{2} B_{2}, \cdots A_{n} B_{n}$, and associated closed curves $\bar{J}_{1}, \bar{J}_{2}, \bar{J}_{3}, \cdots \bar{J}_{n}$, such that

(1) $A_{i} B_{i}$ contains a segment in common with $A_{i-1} B_{i-1} \dagger$ and a segment in common with $A_{i+1} B_{i+1}$, but contains no point in common with any other $A_{j} B_{j}$,

(2) the curves $\bar{J}_{k}$ and $J$ contain only the arc $A_{k} B_{k}$ in common,

(3) the interior of $\bar{J}_{k}$ is without $J$,

(4) neither $\bar{J}_{k}$ nor its interior contains any point of the set $K$.

* A point-set $M$ is said to lie between two closed curves if one of these curves lies within the other one and $M$ lies without the first one and within the second one.

$\dagger$ It is understood throughout this argument that the subscripts of the $A$ 's and $B$ 's are to be reduced modulo $n$.

Trans. Am. Math. Soc. 11 
Let $\bar{G}$ denote the set of curves $\bar{J}_{1}, \bar{J}_{2}, \bar{J}_{3}, \ldots \bar{J}_{n}$ and let $U$ denote the set of $\operatorname{arcs} A_{1} B_{1}, A_{2} B_{2}, \cdots A_{n} B_{n}$. The arc $A_{k} B_{k}$ contains a point $O_{k}$ which belongs to no other arc of the set $U$. By Theorem 44 there exists a closed curve $C_{k}$, containing $O_{k}$, such that every point of $C_{k}$ belongs to some curve of the set $\bar{G}$ and such that every point within $C_{k}$ is within $\bar{J}_{k}$ but without every other curve of the set $\bar{G}$. The $\operatorname{arcs} A_{k} B_{k}$ and $A_{k+1} B_{k+1}$ have in common an $\operatorname{arc} A_{k+1} P_{k} B_{k}$. By Theorem 40 there exists an arc $A_{k} \bar{P}_{k} B_{k}$ such that $A_{k} \bar{P}_{k} B_{k}$ is a subset of $I$ (the interior of $J$ ). The point-set $\left(\bar{J}_{k}-A_{k} \overline{\left.O_{k} B_{k}\right)}+A_{k} \bar{P}_{k} B_{k}\right.$ is a closed curve containing in its interior the point $P_{k}$ of the closed curve $\bar{J}_{k+1}$. With the assistance first of Theorem 43 and afterwards of Theorem 44 it may be easily proved that there exists a closed curve $\bar{C}_{k}$, containing $P_{k}$, such that (1) every point of $\bar{C}_{k}$ belongs to some curve of the set $\bar{G},(2)$ every point within $\bar{C}_{k}$ is within $\bar{J}_{k}$ and within $\bar{J}_{k+1}$ but without every other curve of the set $\bar{G}$. It is clear that the curves $C_{k}$ and $J$ have only the arc $B_{k-1} O_{k} A_{k+1}$ in common while the curves $\bar{C}_{k}$ and $J$ have only the arc $A_{k+1} P_{k} B_{k}$ in common. It is clear furthermore that $C_{k}$ and $\bar{C}_{k}$ have in common an arc $D_{k} A_{k+1}$ which has no point except $A_{k+1}$ in common with $J$. The curves $C_{k}$ and $\bar{C}_{k-1}$ have in common an arc $E_{k} B_{k-1}$ which has no point except $B_{k-1}$ in common with $J$. By Theorem 40 there exist $\operatorname{arcs} E_{k} D_{k}$ and $D_{k} E_{k+1}$ such that $E_{k} D_{k}$ lies within $C_{k}$ and $D_{k} E_{k+1}$ lies within $\bar{C}_{k}$. Let $J_{1}$ denote the point-set

$$
E_{1} D_{1}+D_{1} E_{2}+E_{2} D_{2}+D_{2} E_{3}+\cdots+E_{n} D_{n}+D_{n} E_{1} .
$$

It can be proved with the help of Theorem 27 that $J_{1}$ is a closed curve enclosing $J$ and such that no point of $K$ is between $J_{1}$ and $J$.

Similarly there exists a closed curve $J_{2}$ lying within $J$ and such that no point of $K$ is between $J$ and $J_{2}$.

TheOREM 47. If the closed curve $J_{1}$ encloses the closed curve $J_{2}$ then the set of all points between $J_{1}$ and $J_{2}$ is connected.

Proof. Suppose $A$ and $B$ are two points between $J_{1}$ and $J_{2}$. Let $R_{2}$ denote the interior of $J_{2}$. By Theorem 46 there exists within $J_{1}$ a closed curve $J$ such that there is no point of the closed point-set $A+B+J_{2}+R_{2}$ between $J_{1}$ and $J$. By Theorem 16 there exists an arc $A X B$ in the exterior of $J_{2}$. The point-set $J+A X B$ contains as a subset an arc $A Y B$ that lies between $J_{1}$ and $J_{2}$. It follows that the set of all points between $J_{1}$ and $J_{2}$ is connected.

TheOREM 48.* Suppose that $K$ is a closed, bounded set of points and that $S-K=S_{1}+S_{2}$ where $S_{1}$ and $S_{2}$ are point-sets such that (1) every two points of $S_{i}(i=1,2)$ can be joined by an arc lying entirely in $S_{i}$, (2) every arc joining

* Cf. A. Schoenflies, Ueber einen grundlegenden Satz der Analysis Situs, N a c h r i c h t e n der Göt tinger Gesellschaft der Wissenschaften, 1902, p. 185. 
a point of $S_{1}$ to a point of $S_{2}$ contains a point of $K,(3)$ if $O$ is a point of $K$ and $P$ is a point not belonging to $K$ then $P$ can be joined to $O$ by an arc that has no point except $O$ in common with $K$. Every point-set $K$ that satisfies these conditions is a simple closed curve.

Theorem 48 can be proved on the basis of the preceding theorems by an argument in large part similar to that employed by Lennes.* He makes use of straight lines and polygons but an argument that is in large part similar can be carried through with the use of arcs and closed curves. Schoenflies uses metrical properties.

Definition. An open curve is a closed, connected set of points $M$ such that if $P$ is a point of $M$ then $M-P$ is the sum of two mutually exclusive connected point-sets, neither of which contains a limit point of the other one. $\dagger$

It is easy to see that every open curve $l$ satisfies Axioms 1-4, $5^{\prime}, 6$, and 8 of my papers The Linear Continuum in Terms of Point and Limit $\ddagger$ and On the Linear Continuum. $\$$ It follows that Theorems 1-11 of I and Theorem $E$ of II hold true on $l$.

TheOREm 49. If $A$ and $B$ are distinct points on the open curve $l$, then the point-set t composed of $A, B$, and the segment $\| A B$ of $l$ is a simple continuous arc from $A$ to $B$.

Proof. Clearly $t$ is closed and connected and contains no connected proper subset that contains $A$ and $B$. Suppose $t$ is not bounded. Then $l$ contains two points $X$ and $Y$ such that if $Z$ is any point on the ray $X Y$ then the segment $X Z$ is not bounded. There exists a closed curve $J$ enclosing $X$. There exists on the ray $X Y$ a countably infinite sequence of points $P_{1}, P_{2}, P_{3}, \cdots$ that has $X$ as a sequential limit point. The segment $X P_{n}$ contains a point without $J$. It follows that it has a point $\bar{P}_{n}$ in common with $J$. There exists, on $J$, a point $O$ which is a limit point of $P_{1}+P_{2}+P_{3}+\cdots$. But it is easy to see that $X$ is the only limit point of $\bar{P}_{1}+\bar{P}_{2}+\bar{P}_{3}+\cdots$. Thus the supposition that $t$ is not bounded leads to a contradiction. Hence $t$ satisfies all the requirements of Lennes' definition of a continuous simple arc from $A$ to $B$.

THEOREM 50. Every ray of an open curve contains a set of points that has no limit point.

Proof. Suppose that every infinite subset of the ray $O A$ of the open curve $l$

* Loc. cit., $\S 5$.

$\dagger$ If $P$ is a point of the open curve $M$ and $M-P$ is the sum of two connected point-sets $M_{1}$ and $M_{2}$, then $M_{1}$ and $M_{2}$ are called rays.

$\ddagger$ Annals of Mathematics, vol. 16 (1915), pp. 123-133. This paper will be referred to as $I$.

\$Bulletin of the American Mathematical Society, vol. 22 (1915), pp. 117-122. This paper will be referred to as II.

॥ For definition of segment see II, p. 120. 
has a limit point. Then the closed set of points $O A+O$ possesses the HeineBorel property. There exists on $l$ a point $D$ in the order $A O D$. If $P$ is a point of the ray $O A$ there exists a point $B$ in the order $O P B$. There exists about $P$ a region $R$ containing no point of $l$ that is not on the segment $D B$. It follows that $O A+O$ is covered by a finite set of regions $R_{1}, R_{2}, R_{3}, \cdots R_{n}$. But for each $R_{n}$ there is a point $B_{n}$ such that every point that $R_{n}$ has in common with $l$ lies between $D$ and $B_{n}$. It follows that there exists on $O A$ a point $E$ such that no region of the set $R_{1}, R_{2}, \cdots R_{n}$ contains any point of $l$ that does not lie between $O$ and $E$. But $O A$ contains points that do not lie between $O$ and $E$. Thus the supposition that $l$ is compact leads to a contradiction.

Theorem 51. If $l$ is an open curve then $S-l=S_{1}+S_{2}$ where $S_{1}$ and $S_{2}$ are connected point-sets such that every arc from a point of $S_{1}$ to a point of $S_{2}$ contains at least one point of $l$.

Proof. There exists on $l$ an arc $\bar{A} O \bar{B}$. There exists about $O$ a region $\bar{R}$ containing neither $\bar{A}$ nor $\bar{C}$. There exists an arc $\bar{A} \bar{X} \bar{B}$ that contains no point of $\bar{R}$. There exist points $A$ and $B$ such that $A$ is the last point that $\vec{A} \vec{X} \bar{B}$ has in common with the ray $O \bar{A}$ while $B$ is the first point following $A$ that $\bar{A} \bar{X} \bar{B}$ has in common with the ray $O B$. The arc $\bar{A} \bar{X} \bar{B}$ contains a segment $A X B$. This segment has no point except $A$ and $B$ in common with $l$.

On $l$ there exist points $\bar{C}$ and $\bar{D}$ in the order $\bar{C} A O B \bar{D}$.

$$
l=\underline{A O B}+\operatorname{ray} B \bar{D}+\operatorname{ray} A \bar{C} .
$$

The ray $A \bar{C}$ is not bounded. Hence it contains at least one point without the curve $A O B X A$. Hence if the ray $A \bar{C}$ contains a point within $A O B X A$ it must contain a point on $A O B X A$. But this is contrary to hypothesis. It follows that the interior of $A O B X A$ contains no point of $l$. About $O$ there is a region which contains no point of $A X B+$ ray $A \bar{C}+$ ray $B \bar{D}$. In this region there is an arc $C Y D$ such that $C$ is on $O A, D$ is on $O B$, and $C Y D$ is entirely without $A O B X A$. By Theorem 40 there exists an arc $C Z \bar{D}$ such that $C Z D$ is within $A O B X A$. The closed curve $C Y D Z C$ contains no point of $l$ except points of $C O D$. The interior of $C Y D Z C=C O D+$ the interior of $C O D Z C+$ the interior of $C O D Y C$. Let $O_{1}$ and $O_{2}$ denote definite points within $C O D Z C$ and within $C O D Y C$ respectively. Let $S_{1}$ denote the set of all points $\left[P_{1}\right]$ such that $P_{1}$ can be joined to $O_{1}$ by an arc containing no point of $l$. Let $S_{2}$ denote the set of all points $\left[P_{2}\right]$ such that $P_{2}$ can be so joined to $O_{2}$. Clearly $S_{1}$ and $S_{2}$ are connected. It remains to show (1) that every arc joining a point of $S_{1}$ to a point of $S_{2}$ contains at least one point of $l,(2)$ that every point of $S-l$ belongs either to $S_{1}$ or to $S_{2}$.

(1) If a point of $S_{1}$ can be joined to a point of $S_{2}$ by an arc that contains no point of $l$ then $O_{1}$ can be joined to $O_{2}$ by such an arc. 
Suppose $O_{1} W O_{2}$ is an arc from $O_{1}$ to $O_{2}$. Then there exist points $\bar{O}_{1}$ and $\bar{O}_{2}$ such that $\bar{O}_{1}$ is the last point that $O_{1} W O_{2}$ has in common with $C Z D$ while $\bar{O}_{2}$ is the first point following $\bar{O}_{1}$ that $O_{1} W O_{2}$ has in common with $C Y D$. The arc $O_{1} W O_{2}$ contains a segment $\bar{O}_{1} \bar{W} \bar{O}_{2}$ which lies entirely without $C Y D Z C$. By Theorem 25 the interior of $C Y D Z C$ lies either within $\bar{O}_{1} \bar{W} \bar{O}_{2} C \bar{O}_{1}$ or within $\bar{O}_{1} \bar{W} \bar{O}_{2} D \bar{O}_{1}$. Suppose it lies within the former. Then the ray $O D$ contains a point $D$ within $\bar{O}_{1} \bar{W} \bar{O}_{2} C \bar{O}_{1}$. Hence it contains a point on $\bar{O}_{1} \bar{W} \bar{O}_{2} C \bar{O}_{1}$. But the arc $\bar{O}_{2} C \bar{O}_{1}$ contains no point of the ray $O D$. Hence $\bar{O}_{1} \bar{W}_{O_{2}}$ must contain a point of this ray.

(2) Suppose $P$ is a point not lying on $l$. There exists an arc $P O$. Let $G$ denote the first point that $P O$ has in common with $l$. There exist (Fig. 8) arcs $K L M$ and $K N M$ having no point in common except $K$ and $M$ and such that $K$ and $M$ are the only points that the closed curve $K L M N K$ has in common with $l$ while the interior of $K L M N K=K G M+$ the interior of $K G M L K+$ the interior of $G K M N K$. It can* be proved that there exist

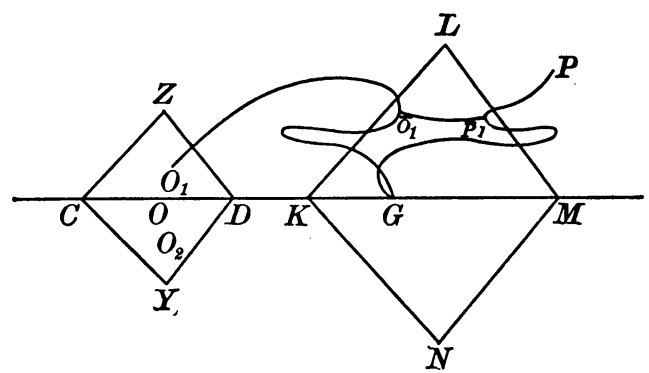

FIG. 8

$\operatorname{arcs} O_{1} G$ and $O_{2} G$ neither of which contains any point except $G$ in common with $l$. The arc $O_{1} G$ contains either a point within $K G M L K$ or a point within $K G M N K$. Suppose it contains a point $\bar{O}_{1}$ within $K G M L K$. Then $\mathrm{O}_{2} \mathrm{G}$ cannot contain a point within $K G M L K$. For if it did then $\mathrm{O}_{1}$ and $\mathrm{O}_{2}$ could be joined by an arc containing no point of $l$. Hence $\mathrm{O}_{2} G$ must contain a point $\bar{O}_{2} \cdot$ within $K G M N K$. But $P G$ must contain a point within $K G M L K$ or a point within $K G M N K$. Suppose it contains a point $\bar{P}_{2}$ within $K G M L K$. Then there exists an arc $\bar{P}_{1} \bar{O}_{1}$ lying entirely within $K G M L K$ and therefore containing no point of $l$. The point-set $P G+\bar{P}_{1} \bar{O}_{1}+O_{1} G$ contains as a subset an arc $P O_{1}$ which contains no point of $l$. Hence in this case $P$ belongs to $S_{1}$. If $P G$ contains a point within $K G M N K$ then $P$ belongs to $S_{2}$. Thus every point of $S-l$ belongs either to $S_{1}$ or to $S_{2}$.

ThEOREM 52. If there exists in $S$ a system of open curves such that through every two points of $S$ there is one and only one curve of this system then there is a one-to-one continuous correspondence between $S$ and an ordinary number plane.

* Cf. proofs of Theorems 46 and 32. 
Theorem 52 may be proved with the assistance of Theorem 51 of the present paper together with results obtained in my paper On a set of postulates which suffice to define a number-plane.*

\section{INDEPENDENCE EXAMPLES}

Let $\Sigma_{1}$ denote the set of Axioms 1-8. In the following, $E_{i} \dagger$ denotes an example of a system in which Axiom $i$ is false but all the other axioms of the set $\Sigma_{1}$ are true. In each example $E_{i}$ use is made of a well-defined space $\delta_{i}$. In every case the points of $E_{i}$ are the ordinary points of $S_{i}$ but the regions of the various $E_{i}$ 's are defined in various ways. For every $i$, except 1 and 8 , $S_{i}$ is an ordinary euclidean space of two dimensions.

$E_{1} . S_{1}$ is the $S_{1}$ described in example $E_{1567}$ of my paper The linear continuum in terms of point and limit. $\ddagger$ The point $\left(x_{1}, y_{1} ; x_{2}, y_{2}\right)$ is a limit point of the point-set $M$ it and only if Condition I§ is fulfilled. Starting with this definition of limit point, one may define the terms arc and closed curve as in $\$ 4$. It can be shown that every such closed curve $J$ divides $S_{1}$ into two subsets $I$ and $E$ such that every infinite set of points in $I$ has at least one limit point. The point set $I$ is called the interior of $J$. Finally a region is defined as the interior of a closed curve.

$E_{2}$. A set of points $M$ is a region if and only if $M$ is either an ordinary Jordan region or the sum of two ordinary Jordan regions $R_{1}$ and $R_{2}$ such that $R_{1}^{\prime}$ and $R_{2}^{\prime}$ have no point in common.

$E_{3}$. A set of points $M$ is a region if and only if $M$ is either an ordinary Jordan region or the set of all points lying between two Jordan curves one of which encloses the other one.

$E_{3}^{\prime} . S_{3}^{\prime}$ is the set of all points on an ordinary straight line. A region is a segment.

$E_{4}$. A set of points $M$ is a region if and only it $M$ is either a half-plane or an ordinary Jordan region.

$E_{5} . S_{5}$ is an ordinary sphere. The point-set $M$ is a region if and only if $M$ is one of the two parts into which $S_{5}$ is divided by an ordinary closed curve.

$E_{6}$. Choose a set of rectangular axes $O X$ and $O Y$. Let $k_{1}$ denote the closed interval from $(0,-1)$ to $(0,1)$ together with the point $(1 / \pi, 0)$ and all points of $y=\sin 1 / x$ that lie between $x=0$ and $x=1 / \pi$. Let $k_{2}$ denote some definite arc that joins the point $(1 / \pi, 0)$ to the point $(0,1)$, contains no point of $y=\sin 1 / x$, except the point $(1 / \pi, 0)$, and lies, except for its endpoints, entirely in Quadrant I. A point-set $M$ is a region if and only if $M$

* These Transactions, vol. 16 (1915), pp. 27-32.

$\dagger$ For Axiom 3 I give two independence examples, $E_{3}$ and $E_{3}^{\prime}$.

$\ddagger$ Loc. cit., page 131.

\& Loc. cit. 
is either an ordinary Jordan region or the set of points enclosed by $k_{1}+k_{2}$.

$E_{7}$. This example is the same as $E_{6}$ except that $k_{2}$ is replaced by an arc from $(1 / \pi, 0)$ to $(0,1)$ that contains no point of $y=\sin 1 / x$, except the point $(1 / \pi, 0)$, and furthermore contains no point in Quadrant I.

$E_{8} . S_{8}$ is an ordinary euclidean space of three dimensions. A set of points is a region if and only if it is the interior of a cube.

\section{ConCERNING $\Sigma_{1}, \Sigma_{2}$, AND $\Sigma_{3}$}

Let $\Sigma_{2}$ denote the set of Axioms $1-5,6^{\prime}, 7^{\prime}, 8$, where $6^{\prime}$ and $7^{\prime}$ are as follows:

Axiom $6^{\prime} .^{*}$ If $R$ is a region and $A B$ is an arc such that $A B-A$ is a subset of $R$ then $(R+A)-A B$ is connected.

Axım $7^{\prime} . \dagger \quad$ Every boundary point of a region is a limit point of the exterior of that region.

Theorem $A$. In a space satisfying $\Sigma_{1}$ every region is the interior of a closed curve.

Theorem $A$ may be proved with the assistance, in particular, of Axioms 6 and 7 and Theorem 48.

Theorems 1-45 are consequences of $\Sigma_{2}$ as well as of $\Sigma_{1}$. It is not true however that in every space satisfying $\Sigma_{2}$ every region is the interior of a simple closed curve. Indeed $\Sigma_{2}$ is satisfied if in an ordinary euclidean plane the term region is applied to every bounded, connected set of points $R$, of connected exterior, such that every point of $R$ is interior to some triangle that lies in $R$. It is easy however to show that though $\Sigma_{1}$ and $\Sigma_{2}$ are not absolutely equivalent, they are equivalent with respect tof point and limit point of a pointset as defined in $\$ 2$.

Let $\Sigma_{3}$ denote the set of Axioms $1^{\prime}, 2^{\prime}, 3,4,5,6^{\prime}, 7^{\prime}$, and 8 , where $1^{\prime}$ and $2^{\prime}$ are as follows:

Axıм 1'. If $P$ is a point, there exists an infinite sequence of regions $R_{1}$, $R_{2}, R_{3}, \cdots$ such that (1) $P$ is the only point they have in common, (2) for every $n, R_{n+1}$ is a proper subset of $R_{n}$, (3) if $R$ is a region about $P$ then there exists $n$ such that $R_{n}^{\prime}$ is a subset of $R$.

Aхгом 2'. Every two points of a region are the extremities of at least one simple continuous arc that lies wholly in that region.

${ }^{*}$ Cf. Theorems 31 and 32.

$\dagger$ Cf. Theorem 20.

$\ddagger$ The statement that $\Sigma_{1}$ and $\Sigma_{2}$ are equivalent with respect to point and limit point as defined in $\$ 2$ signifies that every statement in terms of point and limit point of a point-set that follows from $\Sigma_{1}$ (together with the above mentioned definition of limit point of a pointset) follows also from $\Sigma_{2}$ (together with that definition) and conversely. The statement that $\Sigma_{1}$ and $\Sigma_{2}$ are not absolutely equivalent signifies that they are not equivalent with respect to point and region, the undefined symbols in terms of which they are both stated. 
Theorems 1-52 are all consequences of $\Sigma_{3}$. Nevertheless there exist spaces (see for instance Example $E_{1}$ of $\S 8$ ) that satisfy $\Sigma_{3}$ but are neither metrical, descriptive, nor separable. If however there be added to $\Sigma_{3}$ the axiom that there exists a system of open curves such that through every two points there is one and only one curve of this system, the resulting set of axioms is potentially metrical and, indeed, is categorical with respect to point and limit point of a point-set. See Theorem 52.

University of Pennsyluania 